\title{
Article
}

\section{The Impacts of Sun Exposure on Worker Physiology and Cognition: Multi-Country Evidence and Interventions}

\author{
Leonidas G. Ioannou ${ }^{1,2}{ }^{\oplus}$, Lydia Tsoutsoubi ${ }^{1}{ }^{\oplus}$, Konstantinos Mantzios $^{1}{ }^{\circledR}$, Giorgos Gkikas ${ }^{1}$, Jacob F. Piil ${ }^{2}(\mathbb{D}$,

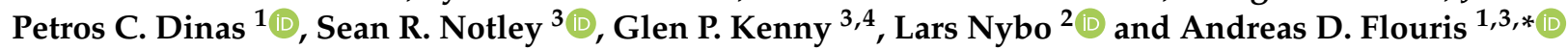 \\ 1 FAME Laboratory, Department of Physical Education and Sport Science, University of Thessaly, \\ 42100 Trikala, Greece; ioannouLG@gmail.com (L.G.I.); lydiatsoutsoubi@gmail.com (L.T.); \\ konstantinosmantzios@gmail.com (K.M.); ggkikas@uth.gr (G.G.); petros.cd@gmail.com (P.C.D.) \\ 2 Department of Nutrition, Exercise and Sports, August Krogh Building, University of Copenhagen, \\ 2100 Copenhagen, Denmark; jpp@nexs.ku.dk (J.F.P.); nybo@nexs.ku.dk (L.N.) \\ 3 Human and Environmental Physiology Research Unit, Faculty of Health Sciences, University of Ottawa, \\ Ottawa, ON K1N 6N5, Canada; snotley@uottawa.ca (S.R.N.); gkenny@uottawa.ca (G.P.K.) \\ 4 Clinical Epidemiology Program, Ottawa Hospital Research Institute, Ottawa, ON K1H 8L6, Canada \\ * Correspondence: andreasflouris@gmail.com; Tel.: +30-2431-047-042
}

\section{check for} updates

Citation: Ioannou, L.G.; Tsoutsoubi, L.; Mantzios, K.; Gkikas, G.; Piil, J.F.; Dinas, P.C.; Notley, S.R.; Kenny, G.P.; Nybo, L.; Flouris, A.D. The Impacts of Sun Exposure on Worker Physiology and Cognition: Multi-Country Evidence and Interventions. Int. J. Environ. Res. Public Health 2021, 18, 7698. https://doi.org/10.3390/ ijerph18147698

Academic Editor: Jeffery Spickett

Received: 5 June 2021

Accepted: 14 July 2021

Published: 20 July 2021

Publisher's Note: MDPI stays neutral with regard to jurisdictional claims in published maps and institutional affiliations.

Copyright: (c) 2021 by the authors. Licensee MDPI, Basel, Switzerland. This article is an open access article distributed under the terms and conditions of the Creative Commons Attribution (CC BY) license (https:// creativecommons.org/licenses/by/ $4.0 /)$.

\begin{abstract}
Background: A set of four case-control $(n=109)$, randomized-controlled $(n=7)$, crosssectional $(n=78)$, and intervention $(n=47)$ studies was conducted across three countries to investigate the effects of sun exposure on worker physiology and cognition. Methods: Physiological, subjective, and cognitive performance data were collected from people working in ambient conditions characterized by the same thermal stress but different solar radiation levels. Results: People working under the sun were more likely to experience dizziness, weakness, and other symptoms of heat strain. These clinical impacts of sun exposure were not accompanied by changes in core body temperature but, instead, were linked with changes in skin temperature. Other physiological responses (heart rate, skin blood flow, and sweat rate) were also increased during sun exposure, while attention and vigilance were reduced by $45 \%$ and $67 \%$, respectively, compared to exposure to a similar thermal stress without sunlight. Light-colored clothes reduced workers' skin temperature by $12-13 \%$ compared to darker-colored clothes. Conclusions: Working under the sun worsens the physiological heat strain experienced and compromises cognitive function, even when the level of heat stress is thought to be the same as being in the shade. Wearing light-colored clothes can limit the physiological heat strain experienced by the body.
\end{abstract}

Keywords: solar radiation; heat; occupational; labor; performance; core temperature; skin temperature; heart rate; skin blood flow; sweat rate

\section{Introduction}

The long-term health effects of sun exposure have been extensively studied, particularly in relation to skin cancer and cataract, but there is little evidence-based knowledge on the acute impacts of sun exposure. For instance, a laboratory study showed marked negative effects of solar radiation on human cognitive performance [1], but we do not know if the heat from the sun can affect the physiology and cognition of people working outdoors [2,3]. The World Health Organization and the International Labor Organization are developing joint methodologies for estimating the associated work-related burden of disease and injury. However, practical and economically feasible protection strategies for people working outdoors have not been investigated at the size and quality needed to draw robust conclusions and recommendations [4].

Agriculture and construction include the vast majority of employees exposed to the sun $[2,3]$ due to the size of these industries, the requirement to work outdoors, and the lack of cost-effective shading solutions for these occupational settings. The associated 
societal and economic impacts are widespread. The agricultural sector alone, employing one-third of the world's labor force [5], is projected to account for $60 \%$ of global working hours lost to heat stress in 10 years from now, whereas construction is expected to account for $19 \%$ of such loss [3]. These estimates translate to 63 million full-time jobs lost across the globe, with an associated monetary loss of US\$ 1.9 trillion in purchasing power parity terms $[3,6]$. While these figures are astounding, they almost certainly underestimate the phenomena that will occur within the next 10 years for two reasons. First, these projections assume that the increase in global mean temperature at the end of the century will not exceed $1.5^{\circ} \mathrm{C}\left(2.7^{\circ} \mathrm{F}\right)$ of pre-industrial levels [3,7]. Unfortunately, it is now clear that global temperature is already $1.0^{\circ} \mathrm{C}\left(1.8^{\circ} \mathrm{F}\right)$ above pre-industrial levels, and it is likely to reach $1.5^{\circ} \mathrm{C}$ between 2030 and 2052 if it continues to increase at the current rate [8]. This climate change is also expected to increase people's exposure to the sun, particularly for those who work outdoors [9]. Second, they assume that work in agriculture and construction is carried out in the shade. However, recent data confirmed that these employees perform the vast majority of their work outdoors and are directly affected by sun exposure, leading to increased heat strain and impaired capacity for manual labor [10-13].

Based on these important knowledge gaps for addressing sun exposure and heat stress emergencies, the goals of this article are to provide health advisors and medical readers with evidence-based information on (i) the effects of sun exposure on worker physiology and cognition and (ii) a practical and economically feasible protection strategy for the most vulnerable individuals.

\section{Materials and Methods}

The goals of this paper are achieved by presenting relevant findings from a series of studies carried out in different industries and countries that include two large field trials, one randomized controlled trial in laboratory settings, and two field interventions. A set of four interconnected studies was conducted as follows:

\subsection{Aims of the Studies}

- Study 1: The effects of solar radiation on the psychophysical stress experienced by workers who perform manual labor in construction and agriculture. The aim of this study was to investigate the effects of solar radiation on human psychophysical stress during actual work shifts in the heat.

- Study 2: The effects of solar radiation on physiological responses and cognitive function at rest and during physical work. The aim of this study was to perform a controlled, laboratory-based evaluation of the effects of solar radiation on human physiological responses and cognitive performance at rest, during physical work, and post-work recovery by comparing indoor (i.e., without solar radiation) and outdoor (i.e., typical mid-day solar radiation) environments characterized by the same thermal stress.

- Study 3: Identifying factors increasing the adverse effects of sun exposure experienced by agriculture and construction workers. The aim of this study was to investigate possible factors exacerbating the effects of sunlight-induced thermal strain by investigating workers' behavioral habits during actual work shifts in occupational settings.

- Study 4: Interventions to mitigate the sunlight-induced heat strain experienced by people who work in agriculture and construction. The aim of this study was to test interventions to mitigate the sunlight-induced heat strain experienced by workers who work in agriculture and construction.

\subsection{Experimental Protocol}

- Study 1: Detailed information on Study 1, including information about the physiological data we collected as well as supplemental tables and figures, is presented in Appendix A. This case-control study involved monitoring 109 experienced and heat acclimatized agriculture and construction workers during four to five consecutive 
full 11-h work shifts. Physiological, subjective, labor, and environmental data were collected throughout the study. Work hours were characterized by the same thermal stress, but different solar radiation levels were isolated to examine if solar radiation levels can independently modify the physiological heat strain experienced by workers.

- Study 2: Detailed information on Study 2, including information about the physiological data we collected as well as supplemental tables and figures, is reported in Appendix B. This single-blinded randomized controlled trial involved tracking seven participants during exposure to four different environmental conditions (two hot $\left(30^{\circ} \mathrm{C}\right.$ wet-bulb globe temperature (WBGT) with and without solar radiation) and two temperate $\left(20^{\circ} \mathrm{C}\right.$ WBGT with and without solar radiation) ambient conditions) allocated in random order. Physiological and subjective data were collected throughout the experiments. This study was conducted to confirm and complement the findings of Study 1 by delineating the physiological and cognitive impacts of sun exposure on people who perform manual work in environments characterized by the same thermal stress but different solar radiation levels.

- Study 3: Detailed information on Study 3 is overviewed in Appendix C. This crosssectional study involved monitoring 78 agriculture workers from seven countries over a period of three months to examine the color of their clothing, a key factor mediating heat exchange, during actual work shifts performed outdoors.

- Study 4: Detailed information on Study 4 is outlined in Appendix D. This intervention study was conducted to investigate if changes in the color of workers' clothing can modify the physiological heat strain experienced by people who work under the sun. The study involved monitoring 47 outdoor workers during two work shifts ("business as usual" and "white clothing" scenarios) characterized by the same thermal stress and solar radiation levels. Physiological, labor, and environmental data were collected to investigate if white clothes can reduce the physiological heat strain experienced by people who work under the sun.

\section{Results}

Sun exposure increases the physiological heat strain experienced by workers and compromises their cognitive function, even when the level of heat stress is thought to be the same as being in the shade. An overview of our findings is presented in Figure 1, while detailed information is provided under the subheading dedicated to each study. 
$=10$ per cent reduction

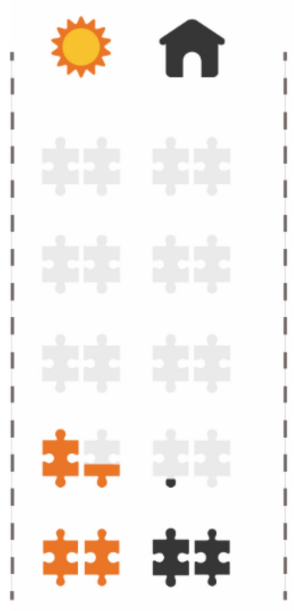

Divided attention

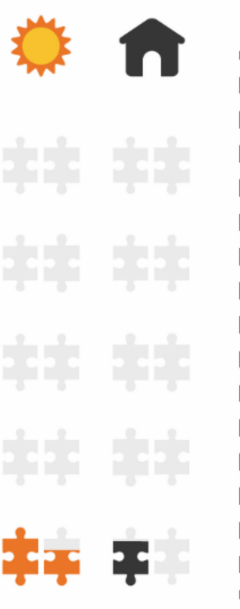

Vigilance

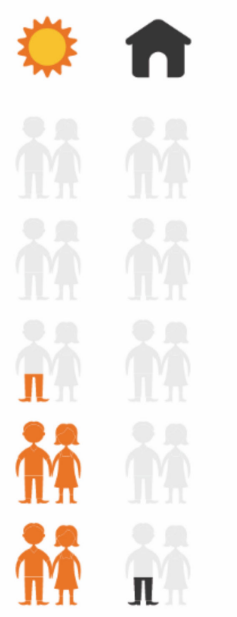

High skin temperature
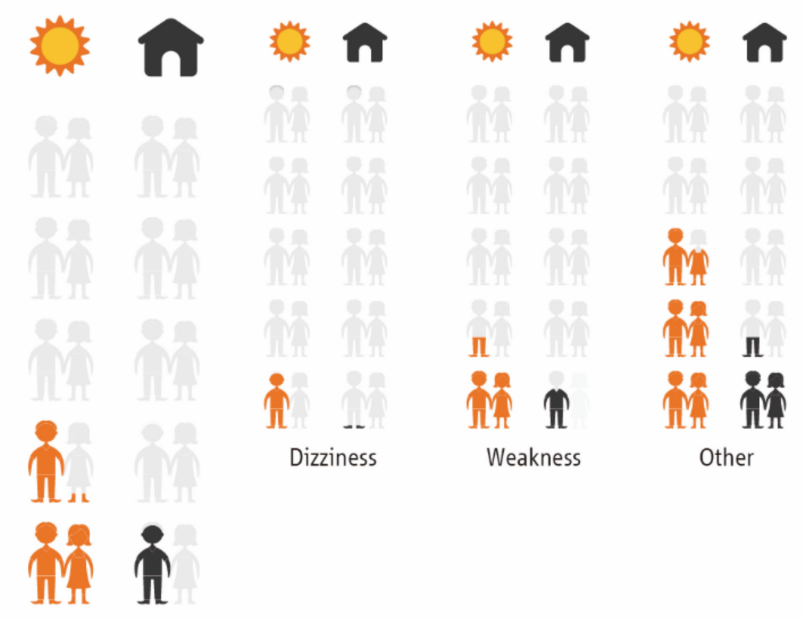

High heat
strain

Figure 1. Cognitive and health impacts while working under the sun (left column) and in the shade (right column) in a hot environment $\left(30^{\circ} \mathrm{C}\right.$ WBGT). Each full-colored puzzle piece indicates a $10 \%$ reduction in cognitive performance (divided attention and vigilance). Each full-colored body figure indicates one-in-ten workers experiencing mean skin temperature higher than $36{ }^{\circ} \mathrm{C}$ (the threshold for progressive symptoms of heat strain [14,15]) or danger-level heat strain, including dizziness, weakness, or other symptoms.

3.1. Results of Study 1: Effects of Solar Radiation on the Psychophysical Stress Experienced by Workers who Perform Manual Labor in Construction and Agriculture

A group of 109 workers (Table A1) participated in the study. During the study, $396 \mathrm{~h}$ (collected from 98 workers) were identified as having equal thermal stress $\left(30^{\circ} \mathrm{C}\right.$ WBGT) but different solar radiation levels (ranging from $0 \mathrm{~W} / \mathrm{m}^{2}$ to $1043 \mathrm{~W} / \mathrm{m}^{2}$ ). Of the 396 h, 108 (27.3\%) were successive hours. Solar radiation levels were positively associated with mean skin temperature $\left(\mathrm{T}_{\mathrm{sk}}\right)(\mathrm{r}=0.419, p<0.001$; Figure 2$)$. This association was characterized by an increase of $\sim 0.2{ }^{\circ} \mathrm{C}$ for every $100 \mathrm{~W} / \mathrm{m}^{2}$ increase in solar radiation levels (Figure 2). On the other hand, a negligible negative association was found between solar radiation levels and core body temperature $\left(\mathrm{T}_{\text {core }}\right)$ as estimated by gastrointestinal temperature ( $\mathrm{r}=-0.141, p=0.035$; Figure A1). Additionally, no associations were identified between solar radiation levels and heart rate (Figure A2) or metabolic rate (Figure A3). Perceived thermal radiation was significantly related to specific items of the Heat Strain Score Index [16] evaluating subjective psychophysical parameters (Table A2). Moreover, significant differences in $\mathrm{T}_{\mathrm{sk}}$ (Figure 2) and labor intensity (Figure A3) were identified between indoor $\left(0\right.$ to $\left.160 \mathrm{~W} / \mathrm{m}^{2}\right)$, mixed $\left(161-320 \mathrm{~W} / \mathrm{m}^{2}\right)$, and outdoor $\left(>320 \mathrm{~W} / \mathrm{m}^{2}\right)$ environments $\left(\mathrm{F}_{(2,360)}=57.791, p<0.001\right)$.

Although we tested the same workers working in environments characterized by the same thermal stress $\left(30{ }^{\circ} \mathrm{C}\right.$ WBGT), having a Heat Strain Score Index greater than 18 (indicating dangerously high risk of experiencing heat strain) was 3.61 times more likely when working outdoors as compared to indoors (Figure 1). Even more so, having a $\mathrm{T}_{\text {sk }}$ above $36{ }^{\circ} \mathrm{C}$ (indicating dangerously high risk of experiencing heat strain $[14,15]$ ) was 10.16 times more likely when working outdoors as compared to indoors (Figure 1). Similarly, the risk for experiencing dizziness, weakness, and other heat strain symptoms (i.e., mild headache, muscle pain, the appearance of red acne, and reduced mental concentration) are $4.44,3.17$, and 2.40 times higher, respectively, when working outdoors compared to indoors (Table A3). 


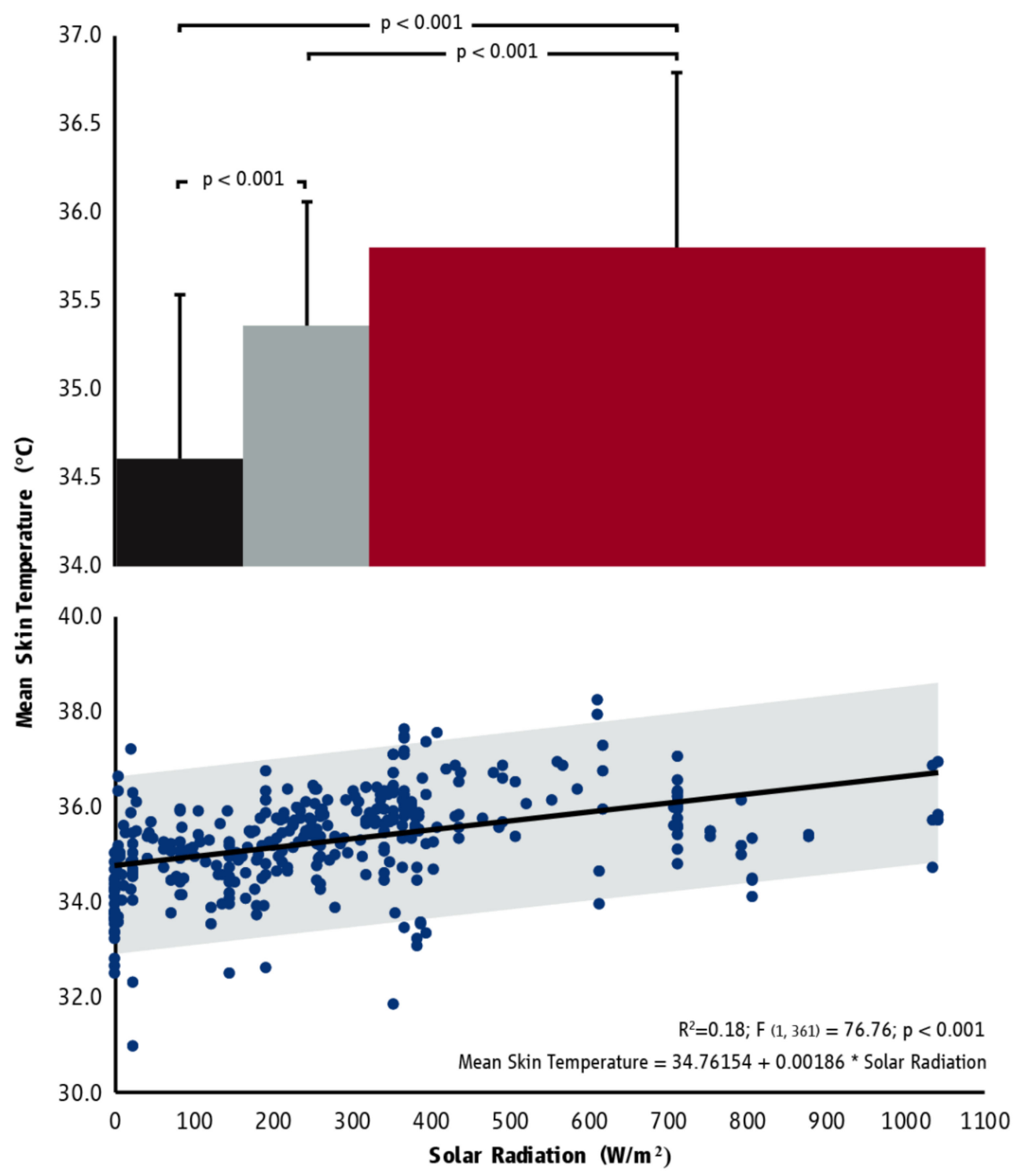

Figure 2. The average $( \pm \mathrm{SD})$ mean skin temperature in indoor (black), mixed (grey), and outdoor (red) environments characterized by the same thermal stress $\left(30{ }^{\circ} \mathrm{C}\right.$ WBGT) but different solar radiation levels (top graph) as well as the association between hourly mean skin temperature and solar radiation (bottom graph). The bar width in the top graph indicates the range of solar radiation of each category corresponding to the horizontal axis, while horizontal brackets indicate statistically significant differences. Shading in the bottom graph corresponds to the $95 \%$ prediction interval.

\subsection{Results of Study 2: Effects of Solar Radiation on Physiological Responses and Cognitive} Function at Rest and during Physical Work

The anthropometric characteristics of the seven volunteers that participated in the study were as follows: age: $22.7 \pm 3.2$ years; body stature: $177.6 \pm 6.1 \mathrm{~cm}$; body mass: $74.3 \pm 8.9 \mathrm{~kg}$; body fat: $20.1 \pm 6.7 \%$; and lean mass: $56.7 \pm 5.1 \%$. Although participants were exposed to environments characterized by equal thermal stress, we identified that exposure to solar radiation had an incremental effect on the mean skin temperature (Figures 3 and 4) of the participants impairing their cognitive performance (Table A5). Furthermore, we found that cognitive performance was positively (i.e., participants made more mistakes when their $\mathrm{T}_{\text {sk }}$ and $\mathrm{T}_{\text {core }}$ were increased) related to $\mathrm{T}_{\text {sk }}$ and $\mathrm{T}_{\text {core }}$ (Table A6). 


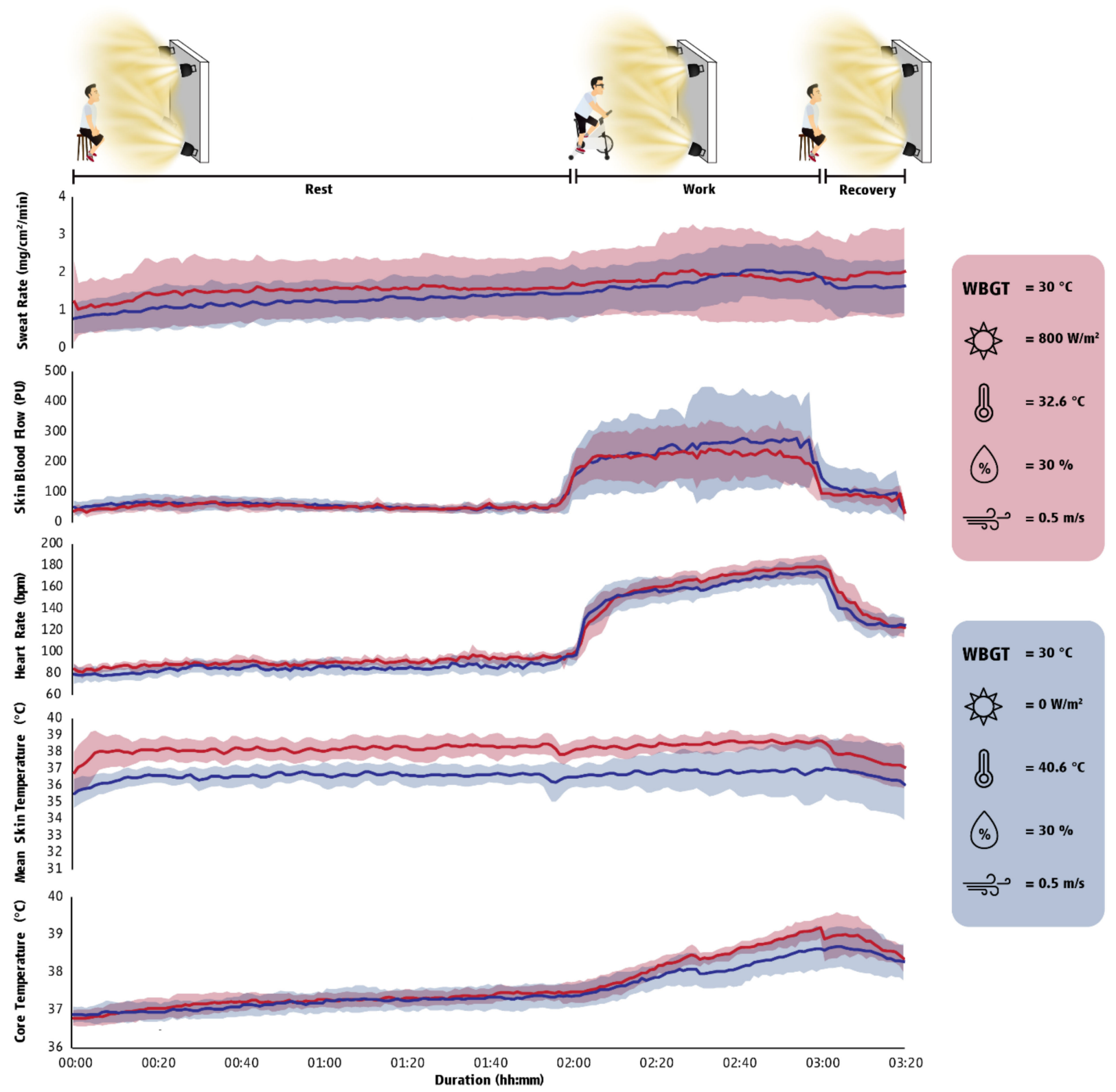

Figure 3. Physiological responses (mean $\pm \mathrm{SD}$ ) during exposure in a hot environment $\left(30^{\circ} \mathrm{C}\right.$ WBGT). The first two hours (00:00 to 02:00) illustrate the fluctuation of physiological responses in resting conditions, the third hour (02:00-03:00) illustrate physiological responses during exercise/work, while the final $20 \mathrm{~min}$ (03:00-03:20) show the responses during recovery time. Red indicates a hot outdoor environment, while blue indicates a hot indoor environment. Sweat rate corresponds to the average sweat rate from the forehead, arm (bicep), and thigh (quadricep) as measured using the ventilated capsule technique, expressed in milligrams per centimeter square per minute. Skin blood flow as measured by laser-Doppler flowmetry corresponds to the average skin blood flow from the forearm (brachioradialis) and leg (gastrocnemius), expressed in perfusion units. Heart rate is expressed in beats per minute. Mean skin temperature estimated from arm, chest, thigh, and leg skin temperatures, expressed in degrees Celsius. Core temperature corresponds to gastrointestinal temperature, expressed in degrees Celsius. Effect sizes for all comparisons between outdoor and indoor environments can be found in Table A5. 

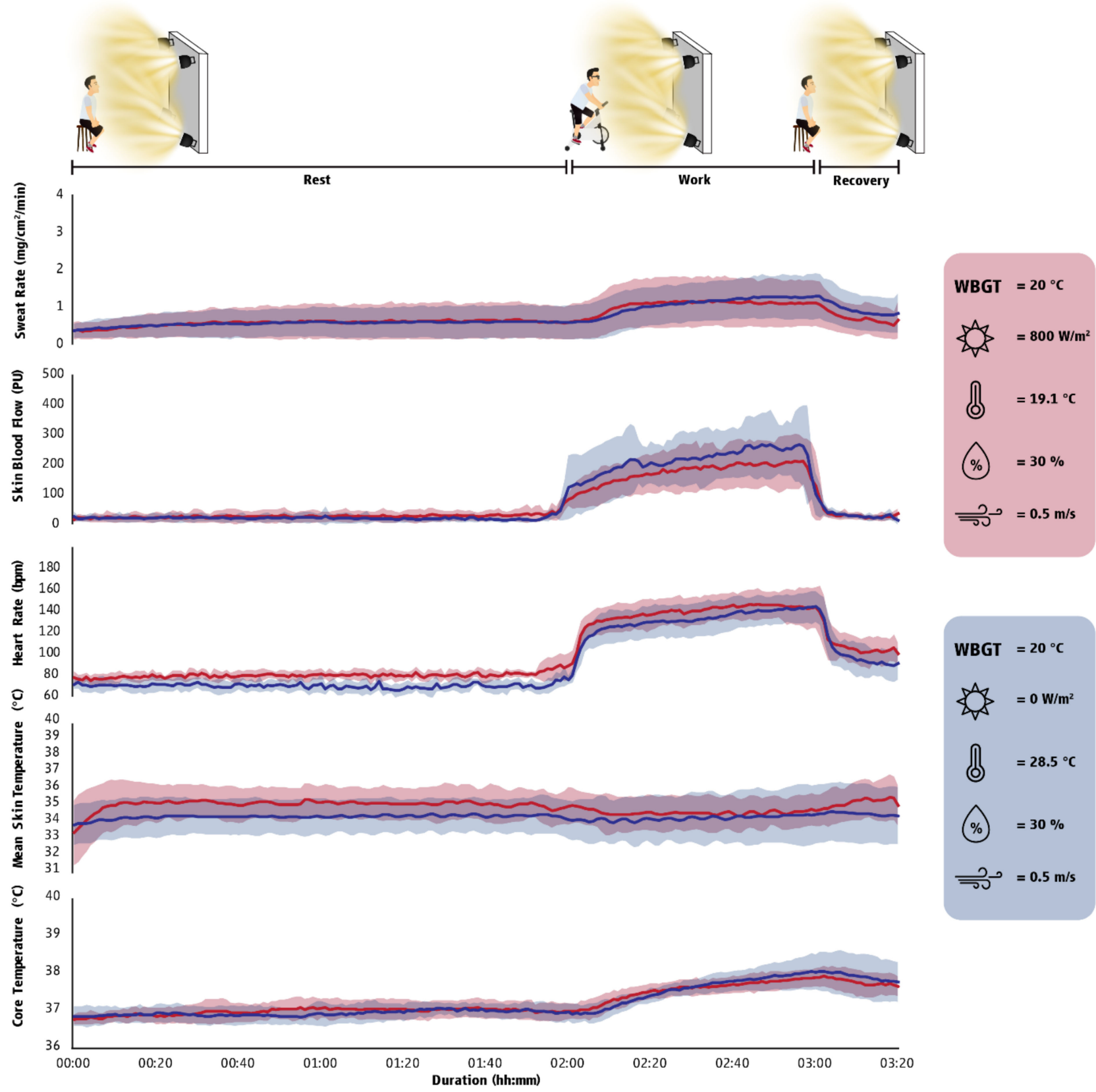

Figure 4. Physiological responses (mean $\pm \mathrm{SD}$ ) during exposure in a temperate environment $\left(20^{\circ} \mathrm{C}\right.$ WBGT). The first two hours (00:00 to 02:00) illustrate the fluctuation of physiological responses in resting conditions, the third hour (02:00-03:00) illustrate physiological responses during exercise/work, while the final $20 \mathrm{~min}$ (03:00-03:20) show the responses during recovery time. Red indicates a thermoneutral outdoor environment, while blue indicates a thermoneutral indoor environment. Sweat rate corresponds to the average sweat rate from the forehead, arm (bicep), and thigh (quadricep) as measured using the ventilated capsule technique, expressed in milligrams per centimeter square per minute. Skin blood flow as measured by laser-Doppler flowmetry corresponds to the average skin blood flow from the forearm (brachioradialis) and leg (gastrocnemius), expressed in perfusion units. Heart rate is expressed in beats per minute. Mean skin temperature estimated from the arm, chest, thigh, and leg skin temperatures, expressed in degrees Celsius. Core temperature corresponds to gastrointestinal temperature, expressed in degrees Celsius. Effect sizes for all comparisons between outdoor and indoor environments can be found in Table A5. 


\subsection{Results of Study 3: Identifying Factors Increasing the Adverse Effects of Sun Exposure Experienced by Agriculture and Construction Workers}

A group of 78 agriculture workers (age: $42.4 \pm 13.0$ years; height: $166.1 \pm 11.5 \mathrm{~cm}$; and weight: $70.5 \pm 19.6 \mathrm{~kg}$ ) was monitored for a total of 112 work shifts over a period of three months. We identified that more than two-thirds $(68.8 \%)$ of the monitored workers wore dark-colored clothes during work under the sun. This was recognized as an important factor increasing the thermal strain experienced by workers. It is important to note that this is against prevailing recommendations and may reflect the lack of knowledge of workers and employers regarding occupational health and safety.

\subsection{Results of Study 4: Interventions to Mitigate the Sunlight-Induced Heat Strain Experienced by Workers Who Work in Agriculture and Construction}

A group of six agriculture (two females) and 41 construction (all males) workers participated in the study (Table A7). The two scenarios ("business as usual" (BAU) and "white clothing" (CLO)) were characterized by similar thermal stress (agriculture: $\sim 23 \pm 4{ }^{\circ} \mathrm{C}$ WBGT; and construction: $\sim 28 \pm 4{ }^{\circ} \mathrm{C}$ WBGT) and solar radiation levels (agriculture: $\sim 920 \pm 300 \mathrm{~W} / \mathrm{m}^{2}$; and construction: $\left.\sim 200 \pm 150 \mathrm{~W} / \mathrm{m}^{2}\right)$. Moreover, task analysis identified that workers performed similar manual work (agriculture (BAU: $194 \pm 54 \mathrm{~W} / \mathrm{m}^{2}$ vs. CLO: $189 \pm 53 \mathrm{~W} / \mathrm{m}^{2}$ ) and construction (BAU: $96 \pm 17 \mathrm{~W} / \mathrm{m}^{2}$ vs. CLO: $86 \pm 17 \mathrm{~W} / \mathrm{m}^{2}$ )) during both scenarios. We identified no significant differences in the $\mathrm{T}_{\text {core }}$ of workers between the tested scenarios (agriculture (BAU: $37.3 \pm 0.3^{\circ} \mathrm{C}$ vs. CLO: $37.2 \pm 0.3^{\circ} \mathrm{C}$ ) and construction: (BAU: $37.3 \pm 0.2{ }^{\circ} \mathrm{C}$ vs. CLO: $37.4 \pm 0.2^{\circ} \mathrm{C}$ )). Importantly, although workers were exposed to environments characterized by the same thermal stress and doing the same labor, workers donning white uniforms experienced a reduced level of physiological heat strain (i.e., small reductions in mean skin temperature and some minor improvement in thermal sensation; Table A8). Furthermore, the average change in $\mathrm{T}_{\mathrm{sk}}$ (from resting conditions) was $13 \%$ and $12 \%$ lower during the white clothing scenario compared to the business-as-usual scenario in agriculture and construction, respectively.

\section{Discussion}

To investigate the physiological and health impacts of sun exposure on workers performing jobs/duties outdoors, a series of four separate but interconnected studies were conducted across different industrial sectors and countries. A large-scale occupational field trial was conducted in Qatar involving 109 construction and agricultural workers. From the collected data, we compared individuals working in the shade versus those working under the sun. We also isolated 396 work hours characterized by the same high level of heat stress $\left(30{ }^{\circ} \mathrm{C}\right.$ WBGT; typical for a Northern hemisphere heatwave) but very different levels of sun exposure: $33 \%$ were in the shade, and $67 \%$ were under the sun. We found that people working under the sun were four times more likely to experience dizziness (i.e., vertigo, presyncope, disequilibrium, or other non-specific feelings), three times more likely to report weakness, and twice more likely to suffer other symptoms of heat strain (i.e., mild headache, muscle pain, the appearance of red acne, and reduced mental concentration), compared to performing the same work in the shade under the same level of heat stress. These clinical impacts of sun exposure were not accompanied by changes in core body temperature but, instead, were linked with changes in skin temperature, which was 10 times more likely to be at levels indicative of heat strain $\left(>36^{\circ} \mathrm{C}\right)$ when working under the sun.

Skin temperature is an important parameter linked with both physiological and psychophysical stress $[14,15,17]$. An increasing number of reports over the last decade have highlighted the importance of high skin temperature as an early indicator of hyperthermia and heat injury, as well as for regulating the intensity of work and exercise [13,17-22]. Recent data from the European Commission project HEAT-SHIELD [23] show that higher skin temperature is linked with reduced capacity to perform manual labor, leading to significant economic losses $[13,24]$. Overall, the findings from the first study show that working under the sun increases skin temperature and the risk for experiencing clinical 
symptoms of heat strain, albeit without markedly altering physiological heat strain as defined by changes in core temperature and heart rate, even in cases where the level of environmental heat stress is considered to be the same as working in the shade. This is probably related to the well-described effect of self-pacing that is known to act proactively to avoid an increase in workers' core body temperature [13,25]. However, self-pacing may not be appropriate when jobs or tasks are time-sensitive, involve productivity incentives, and/or involve workers who are not well trained in their job $[25,26]$.

To delve deeper into the physiological and cognitive impacts of sun exposure, as well as to better position the findings of our field experiments, we conducted in Greece a randomized controlled trial wherein seven healthy individuals were monitored during rest, moderate-intensity physical work, and post-work recovery inside a climate-controlled chamber. We compared values when participants were under the sun versus in the shade in temperate $\left(20^{\circ} \mathrm{C}\right.$ WBGT $)$ and hot $\left(30^{\circ} \mathrm{C}\right.$ WBGT $)$ ambient conditions. This study confirmed that sun exposure could elevate skin temperature without affecting core body temperature. Other physiological responses (heart rate, skin blood flow, and sweat rate) were also increased during sun exposure but to a smaller degree. More importantly, sun exposure reduced cognitive performance in both temperate and hot conditions, leading to a $45 \%$ reduction in divided attention (e.g., auditory and visual stimuli in parallel) and a $67 \%$ reduction in vigilance tasks (see Appendix B for measurement details). Literature suggests that environmental heat stress increases the risk of occupational injuries by promoting fatigue, reduced psychomotor performance, loss of concentration, and reduced alertness [27]. In total, the findings from the second study confirmed that sun exposure generates symptoms of heat strain, such as dizziness and weakness, and it also undermines cognitive function in both temperate and hot conditions, even when the level of heat stress is thought to be the same as being in the shade.

A practical and cost-effective strategy to limit the impact of sun exposure is to change the color of the clothing worn. Wearing white or light-colored clothes increases the reflection of heat from the sun [28-32] and can limit the heat strain (e.g., skin temperature, heart rate, and sweat rate) that the human body experiences during physical work in a hot environment $[29,31]$. Therefore, several heat stress guidelines recommend wearing light-colored clothes during work or exercise under the sun [30,33-36].

To assess attitudes of outdoor workers in relation to the choice of color of their clothing, we monitored 78 workers originating from seven countries (Bangladesh, Cyprus, Egypt, India, Philippines, Romania, and Vietnam) during 112 full work shifts performed outdoors in Cyprus during summer and autumn. Overall, the findings from the third study showed that $68.8 \%$ of the studied outdoor workers wore dark-colored clothes. This is against prevailing recommendations and reflects the lack of education and training on heat-related aspects of occupational health and safety for both workers and employers. Dark-colored clothes have high absorbance of radiative heat, and it is likely that workers would benefit from changing to light-colored work uniforms to minimize the adverse effects of sun exposure.

In an occupational field intervention study performed in Cyprus, we tested whether white clothes can be used as a practical and economically feasible strategy to limit the impacts of sun exposure in agricultural workers. We monitored six workers during two full work shifts characterized by temperate conditions. In the "business as usual" scenario, workers wore their preferred clothes. As expected, based on the results of the abovedescribed observational study, these clothes were mostly black or dark-colored t-shirts and pants. In the "white clothes" scenario, workers were provided with white hats and t-shirts (all 100\% cotton; total cost: US\$ 8.80) and were instructed to wear light-colored pants of their own. The findings from the fourth study demonstrated that the change to white/light-colored clothing minimized the increase in mean skin temperature during work by $13 \%$ (corresponding to a reduction of $0.4^{\circ} \mathrm{C}$ ), despite that the participants worked at the same level of effort in the same outdoor conditions. 
In a second occupational field intervention performed as part of our above-mentioned occupational field trial in Qatar [11], we tested the efficacy of wearing white coveralls for limiting the impacts of sun exposure in construction workers. We monitored 41 construction workers during two full work shifts characterized by moderate heat stress. In the "business as usual" scenario, workers wore their typical work coveralls (dark blue color in most cases) made of polycotton. In the "white coveralls" scenario, workers were provided with white coveralls (total cost: US\$ 6.00) made of cotton or polycotton. This change to white coveralls minimized the increase in mean skin temperature during work by $12 \%$ (corresponding to a reduction of $0.2{ }^{\circ} \mathrm{C}$ ), despite that the participants worked at the same level of effort in the same outdoor conditions. Taken together, these results support existing recommendations for wearing light-colored clothes during work or exercise under the sun [30,33-36] and further demonstrate their practicality and cost-effectiveness in occupational settings. While white work coveralls can be effective in reducing the physiological strain during work under the sun, the adoption of other heat mitigation strategies, such as hydration protocols, work-rest cycles for jobs that do not allow for self-pacing, ventilated garments, and mechanization of heavy work, can further reduce the physiological strain experienced by people working manually outdoors [25].

\section{Conclusions}

Medical staff are often asked whether occupational injuries are caused by exposure to the sun per se or by other parameters of heat stress such as high temperature or humidity. The field studies and laboratory-based clinical trials that we conducted in different parts of the world under both temperate and hot conditions show that working under the sun worsens the physiological heat strain experienced and compromises cognitive function, even when the level of heat stress is thought to be the same as being in the shade. To limit these detrimental impacts of sun exposure in cases where no other cost-effective shading solutions are available, medical staff, as well as health and safety professionals, should advise outdoor workers to wear white or light-colored clothes and hats/helmets. These pale colors increase the reflection of heat from the sun and can limit the heat strain experienced by the body. This multi-country series of field, clinical, and intervention studies raises another important issue; guidelines and policies should consider sun exposure as an important modifier of occupational and public health, not as one mere physical element to be included in the calculation of heat stress. Occupational and public health guidelines should be adapted based on exposure to solar radiation, fueled by a muchneeded estimation of the associated burden of disease and injury.

Author Contributions: Conceptualization, L.G.I., L.N. and A.D.F.; methodology, L.G.I., J.F.P., S.R.N., G.P.K., L.N. and A.D.F.; software, L.G.I. and A.D.F.; validation, L.G.I. and A.D.F.; formal analysis, L.G.I. and A.D.F.; investigation, L.G.I., L.T., K.M., G.G., P.C.D. and A.D.F.; resources, L.N. and A.D.F.; data curation, L.G.I. and A.D.F.; writing - original draft preparation, L.G.I. and A.D.F.; writingreview and editing, L.G.I., L.T., K.M., J.F.P., P.C.D., S.R.N., G.P.K., L.N. and A.D.F.; visualization, L.G.I. and A.D.F.; supervision, A.D.F.; project administration, A.D.F.; funding acquisition, A.D.F. All authors have read and agreed to the published version of the manuscript.

Funding: The study has received funding from the International Labour Organization (Contract Number: 40262271/1) as well as from the European Union's Horizon 2020 research and innovation programme (project HEAT-SHIELD; Grant agreement No 668786).

Institutional Review Board Statement: The study was conducted according to the guidelines of the Declaration of Helsinki and approved by the Bioethical Committee of School of Exercise Science of the University of Thessaly (no. 1217, 5 April 2017; and no. 1303, 6 December 2017) and the National Bioethical Review Board of Cyprus (no. EEBK EP 2017.01.61, 11 May 2017).

Informed Consent Statement: Informed consent was obtained from all subjects involved in the study.

Acknowledgments: We wish to thank the Qatar Ministry of Administrative Development, Labour and Social Affairs, as well as the Supreme Committee for Delivery and Legacy for their organizational support throughout the studies conducted in Qatar. 
Conflicts of Interest: The authors declare no conflict of interest.

\section{Appendix A}

Study 1. Effects of solar radiation on the psychophysical stress experienced by workers who perform manual labor in construction and agriculture.

\section{Appendix A.1. Experimental Protocol}

The experimental protocol (ClinicalTrials.gov (accessed on 1 July 2019) ID: NCT04160728) for these field experiments was approved by the Bioethical Committee of the School of Exercise Science of the University of Thessaly (protocol number: 1217) and the National Bioethical Review Board of Cyprus (protocol number: EEBK EP 2017.01.61) in accordance with the latest Declaration of Helsinki, except for registration in a database. The study involved monitoring 109 experienced (work experience: $4.8 \pm 5.5$ years) and acclimatized (living in the area for more than two months) agriculture and construction workers during four or five consecutive full work shifts. The construction work shifts monitored were (i) 00:00-11:00, (ii) 06:00-17:00, and (iii) 15:30-02:30, covering the entire day. In agriculture, the work shift monitored was 04:00-11:00. Prior to their participation in the study, written informed consent was obtained from all volunteers after a detailed explanation of all the procedures involved.

Self-reported age, body stature, and body mass were collected prior to the experiment. During the field study, continuous heart rate $(\mathrm{HR})$, body core temperature $\left(\mathrm{T}_{\text {core }}\right)$, and mean skin temperature $\left(\mathrm{T}_{\mathrm{sk}}\right)$ data were collected using wireless heart rate monitors (Polar Team2, Polar Electro Oy, Kempele, Finland), telemetric capsules (BodyCap, Caen, France), and wireless thermistors (iButtons type DS1921H, Maxim/Dallas Semiconductor Corp., Sunnyvale, CA, USA), respectively. Skin temperature data were collected from four body sites (chest, arm, thigh, and calf) and were expressed as a weighted average $\left(\mathrm{T}_{\mathrm{sk}}=(0.3\right.$ (chest + arm $)+0.2($ thigh + calf))) [37]. Furthermore, continuous environmental data (air temperature $\left({ }^{\circ} \mathrm{C}\right)$, globe temperature $\left({ }^{\circ} \mathrm{C}\right)$, relative humidity $(\%)$, and air velocity $\left.(\mathrm{m} / \mathrm{s})\right)$ were collected using portable weather stations (Kestrel 5400FW, Nielsen-Kellerman, Boothwyn, PA, USA) installed in close proximity to the workers. Thereafter, these environmental data were utilized to compute solar radiation (using air temperature, relative humidity, globe temperature, and air velocity) by a very well-known iterative method [38].

Real-time task analysis was utilized to evaluate labor intensity. This is a method based on the well-accepted time-motion analysis used to evaluate workers' labor effort $[13,39]$. The main difference between the two methods is that real-time task analysis is performed live at the work site, while time-motion analysis is based on video recordings taken at the work site and analyzed at a later time. Work intensity (i.e., rest, low/medium/highintensity) was based on the International Standard 8996 for the determination of metabolic rate [40]. Specifically, rest $\left(65 \mathrm{~W} / \mathrm{m}^{2}\right)$ was characterized as any activity involving resting and /or sitting at ease. Low-intensity labor $\left(100 \mathrm{~W} / \mathrm{m}^{2}\right)$ included activities incorporating "hand and arm work" or "hand and leg work" such as driving vehicles in normal conditions, machining, and casual walking (at a speed up to $2.5 \mathrm{~km} / \mathrm{h}$ ). Moderate-intensity labor $\left(165 \mathrm{~W} / \mathrm{m}^{2}\right)$ included activities involving "hand and arm work" or "arm and leg work" or "arm and trunk work" such as working with construction equipment, weeding, picking fruits, or walking at a speed between $2.5 \mathrm{~km} / \mathrm{h}$ to $5.5 \mathrm{~km} / \mathrm{h}$. High-intensity labor $\left(230 \mathrm{~W} / \mathrm{m}^{2}\right)$ included any activity involving intense arm and trunk work, carrying heavy material, pushing or pulling heavily, or walking at a speed ranging between $5.5 \mathrm{~km} / \mathrm{h}$ and $7 \mathrm{~km} / \mathrm{h}$.

The raw data collected were used to calculate hourly mean values. Thereafter, these averages were used to isolate work hours characterized by the same thermal stress but different solar radiation levels. Specifically, we isolated work hours characterized by $30^{\circ} \mathrm{C}$ wet-bulb globe temperature (WBGT) rounded at $0.5^{\circ} \mathrm{C}$. At the end of each work shift, self-reported information regarding the psychophysical strain experienced throughout the work shift was collected using the Heat Strain Score Index [16]. A score beyond 18 in this 
tool indicates that a worker has a dangerously high risk of experiencing excessive heat strain [16].

We chose to use WBGT to describe thermal stress because it is the most widely used thermal index $(10,900$ times referred to or used according to Google Scholar metrics (15 October 2019)). Furthermore, it has been specifically designed for work activity assessments and adopted by reputable organizations in occupational safety and health worldwide, including the International Labour Organization [3]. It also incorporates all four environmental parameters (air temperature, relative humidity, wind speed, and solar radiation) for assessing the thermal stress experienced by workers who work in agriculture and construction.

\section{Appendix A.2. Perceived Thermal Radiation Scale}

Alongside the aforementioned questionnaires, perceived thermal radiation was assessed using the following scale:

0 . Non-detectable

1. Detectable

2. Very low

3. Low

4. Somewhat low

5. Neither low nor high

6. Somewhat high

7. High

8. Very high

9. Almost extreme

10. Extreme

\section{Appendix A.3. Statistical Analysis}

Pearson's correlation coefficient (r) was used to examine the relationships between solar radiation, physiological strain $\left(\mathrm{HR}, \mathrm{T}_{\text {core, }}\right.$ and $\mathrm{T}_{\mathrm{sk}}$ ) and labor intensity collected during the study. Similarly, Pearson's correlation coefficient was used to investigate the relationships between perceived thermal radiation scale and subjective psychophysical parameters derived from the Heat Strain Score Index tool. A Bonferroni adjusted alpha of 0.003 was set for detecting statistically significant associations for this set of correlation analyses. Linear regression analyses with prediction intervals were used to examine if solar radiation levels can predict the physiological strain ( $\mathrm{HR}, \mathrm{T}_{\text {core, }}$ and $\left.\mathrm{T}_{\mathrm{sk}}\right)$ and labor intensity. Thereafter, solar radiation was categorized into three groups (indoor (0 to $\left.160 \mathrm{~W} / \mathrm{m}^{2}\right)$, mixed $\left(161\right.$ to $\left.320 \mathrm{~W} / \mathrm{m}^{2}\right)$, and outdoor $\left(>320 \mathrm{~W} / \mathrm{m}^{2}\right)$ environments) based on previous studies $[41,42]$ reporting that $93-97 \%$ of the indoor environments are characterized by solar radiation levels ranging between 0 and $160 \mathrm{~W} / \mathrm{m}^{2}$. We used solar radiation ranging between 161 and $320 \mathrm{~W} / \mathrm{m}^{2}$ (twice the indoor environment) to define mixed environments (workers spending time both indoor and outdoor). Finally, solar radiation levels higher than $320 \mathrm{~W} / \mathrm{m}^{2}$ were defined as outdoor environments. Thereafter, one-way ANOVA with post hoc (LSD) analysis was used to detect potential differences in HR, $\mathrm{T}_{\text {core, }}$ $\mathrm{T}_{\mathrm{sk}}$, and labor intensity between the three solar radiation categories (indoor, mixed, and outdoor environments).

Relative risks were calculated to investigate the risk of experiencing heat-related symptoms in indoor $\left(0-160 \mathrm{~W} / \mathrm{m}^{2}\right)$ and outdoor $\left(>160 \mathrm{~W} / \mathrm{m}^{2}\right)$ conditions. We considered $\mathrm{T}_{\mathrm{sk}}$ values greater than $36^{\circ} \mathrm{C}$ as indicative of high heat strain. This was based on previous studies reporting that the odds of progressive symptoms of heat strain increase rapidly after skin temperature reaches $36^{\circ} \mathrm{C}[14,15]$, leading to a decreased capacity for physical work [43-45]. Thereafter, relative risks were calculated to investigate the probability that a worker has a dangerously high risk of experiencing high heat strain (based on a Heat Strain Score Index greater than 18 or $\mathrm{T}_{\mathrm{sk}}$ above $36^{\circ} \mathrm{C}$ ) in indoor and outdoor conditions. The relative risks, their standard error, and $95 \%$ confidence interval were calculated based on 
a method described by Altman [46]. According to standard methodology [47], nil values were converted to 0.5 to avoid problems with the computation of effects or standard errors. The statistical significances of the identified relative risks values were determined by their accompanied $95 \%$ confidence interval [48].

Statistical analyses were conducted using both the SPSS v25.0 (IBM, Armonk, NY, USA) and Excel spreadsheets (Microsoft Office, Microsoft Corp., Redmond, WA, USA). The level of significance for these analyses was set at $p<0.05$ unless otherwise specified.

Appendix A.4. Results

Table A1. Anthropometric characteristics in Study 1.

\begin{tabular}{lcccc}
\hline & Mean & SD & Minimum & Maximum \\
\hline Age (years) & 33.6 & 8.2 & 18.0 & 56.0 \\
Body mass $(\mathrm{kg})$ & 65.6 & 8.4 & 45.9 & 90.0 \\
Body stature $(\mathrm{m})$ & 1.7 & 0.1 & 1.5 & 1.8 \\
Body mass index $\left(\mathrm{kg} / \mathrm{m}^{2}\right)$ & 23.7 & 3.0 & 17.3 & 33.4 \\
\hline
\end{tabular}

Table A2. Association between "the perceived thermal radiation" and subjective psychophysical parameters derived from the Heat Strain Score Index. * indicates statistically significant association at a Bonferroni adjusted alpha of 0.003 .

\begin{tabular}{|c|c|c|c|}
\hline No. & Item & $\mathbf{r}$ & $p$ \\
\hline 1 & Heat Strain Score Index & 0.517 & $<0.001 *$ \\
\hline 2 & $\begin{array}{l}\text { How would you describe the surface temperature of the } \\
\text { surrounding equipment on your job site today? }\end{array}$ & 0.430 & $<0.001$ * \\
\hline 3 & How much do you feel you sweat today while working? & 0.340 & $<0.001 *$ \\
\hline 4 & How fatigued do you feel during today's work shift? & 0.468 & $<0.001 *$ \\
\hline 5 & How thirsty do you feel during today's work shift? & 0.376 & $<0.001^{*}$ \\
\hline 6 & $\begin{array}{l}\text { How much did the heat affect your ability to perform your } \\
\text { job today? }\end{array}$ & 0.356 & $<0.001 *$ \\
\hline
\end{tabular}

Table A3. Differences in psychophysical parameters between indoor and outdoor workers.

\begin{tabular}{lccc}
\hline Symptom & Relative Risk & $\mathbf{- 9 5 \% ~ C I ~}$ & $\mathbf{+ 9 5 \% ~ C I ~}$ \\
\hline Heat Strain Score Index (danger level) & 3.61 & 2.12 & 6.17 \\
Mean skin temperature above $36{ }^{\circ} \mathrm{C}$ & 10.16 & 4.25 & 24.33 \\
Dizziness & 4.44 & 0.61 & 1.34 \\
Weakness & 3.17 & 1.76 & 5.71 \\
Any heat strain symptom & 2.40 & 1.78 & 3.24 \\
\hline
\end{tabular}




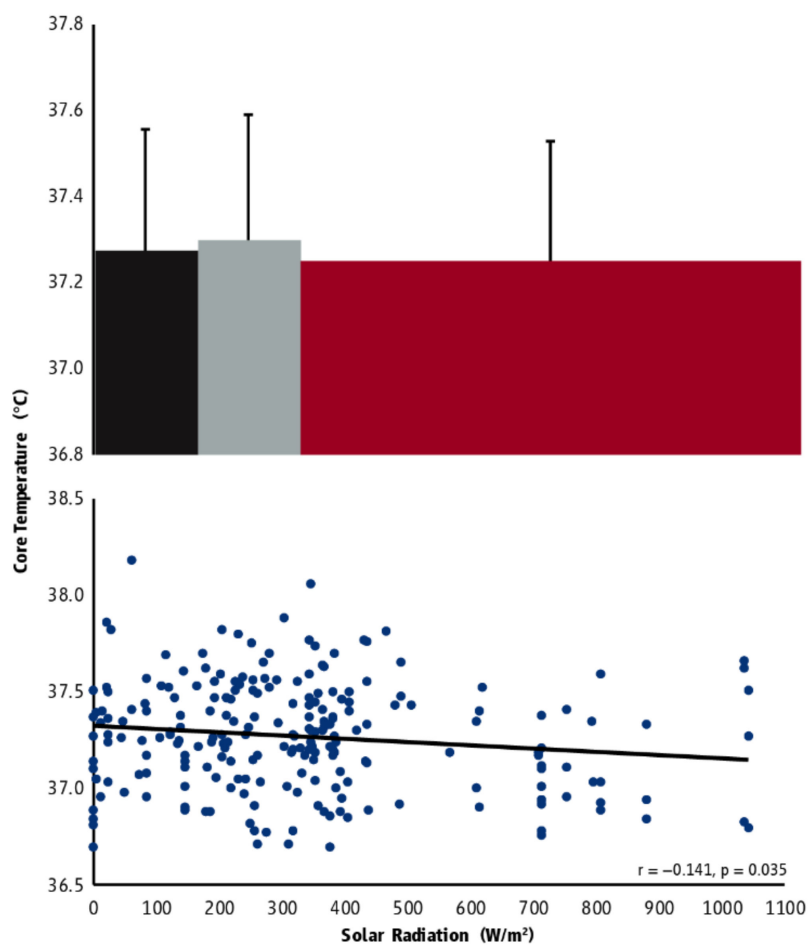

Figure A1. The average $( \pm S D)$ core temperature in indoor (black), mixed (grey), and outdoor (red) environments characterized by the same thermal stress (top graph) as well as the association between hourly core temperature and solar radiation (bottom graph). Bar width in the top graph indicates the range of solar radiation of each category corresponding to the horizontal axis.

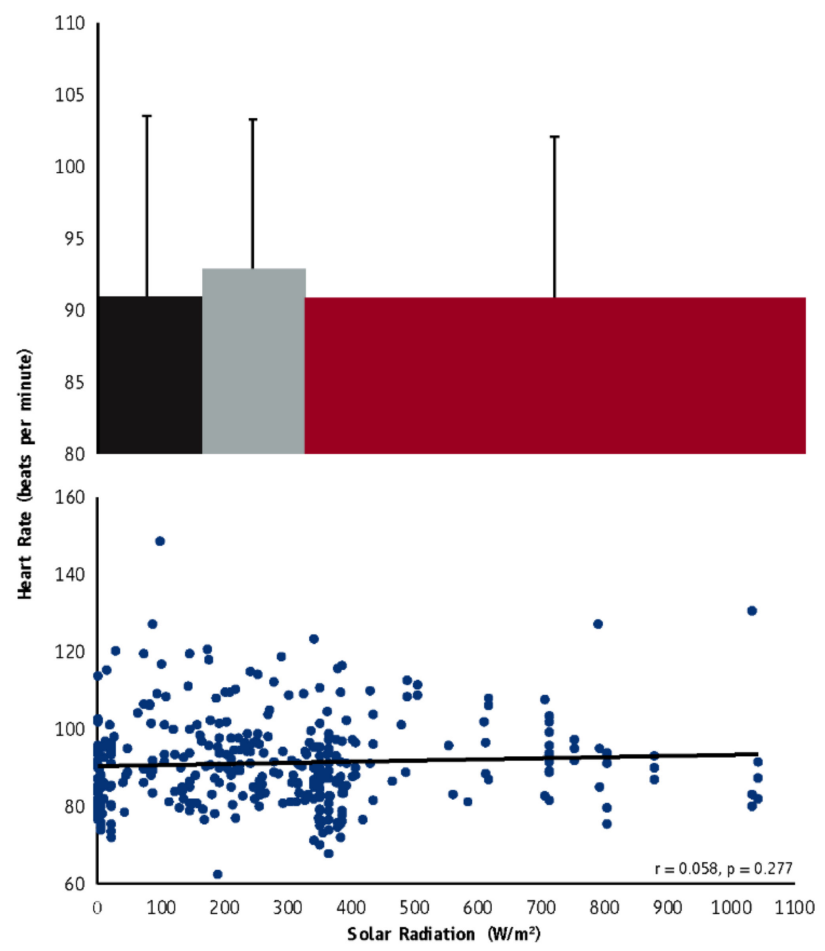

Figure A2. The average ( $\pm \mathrm{SD}$ ) heart rate in indoor (black), mixed (grey), and outdoor (red) environments characterized by the same thermal stress (top graph) as well as the association between hourly heart rate and solar radiation (bottom graph). Bar width in the top graph indicates the range of solar radiation of each category corresponding to the horizontal axis. 


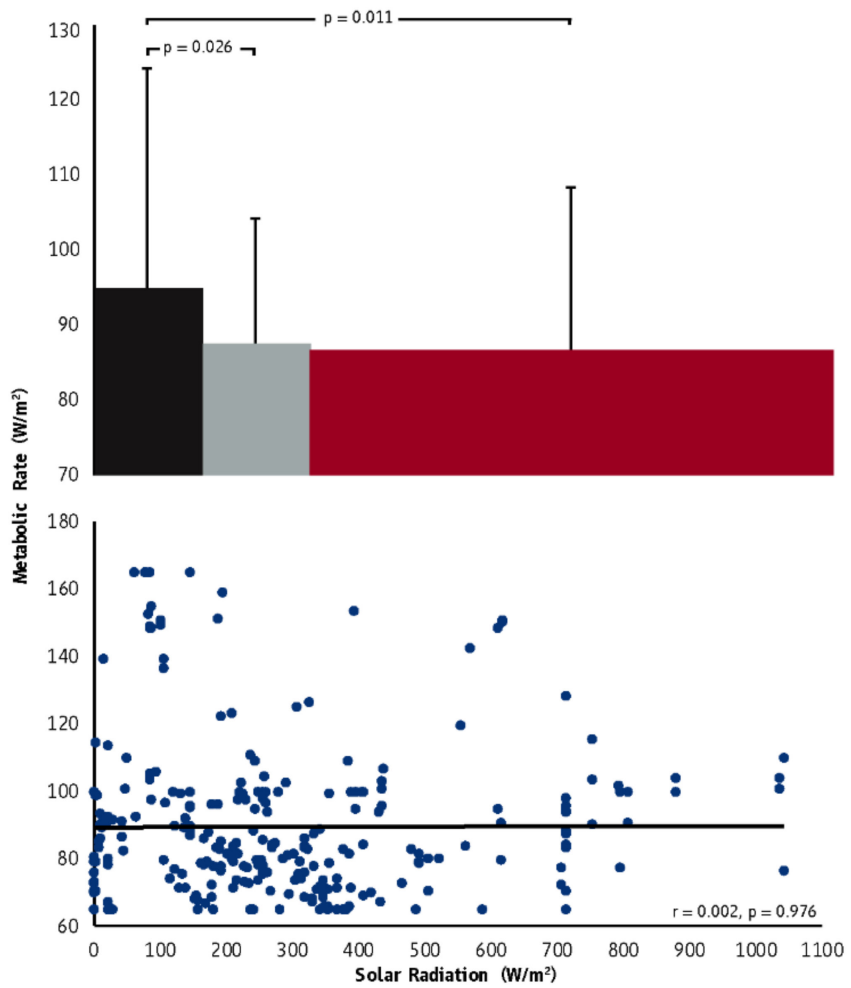

Figure A3. The average $( \pm \mathrm{SD})$ metabolic rate in indoor (black), mixed (grey), and outdoor (red) environments characterized by the same thermal stress (top graph) as well as the association between hourly metabolic rate and solar radiation (bottom graph). Bar width in the top graph indicates the range of solar radiation of each category corresponding to the horizontal axis, while horizontal brackets indicate statistically significant differences.

\section{Appendix B}

Study 2. Effects of solar radiation on physiological responses and cognitive function at rest and during physical work.

\section{Appendix B.1. Experimental Protocol}

This was a single-blind randomized controlled trial (ClinicalTrials.gov (accessed on 1 July 2019) ID: NCT04160741). The experimental protocol was approved by the Bioethical Committee of the School of Exercise Science of the University of Thessaly (protocol number: 1303) in accordance with the Declaration of Helsinki. Seven healthy non-smoking males (see "Sample Size Calculation") participated in the experiments. Prior to their participation in the study, written informed consent was obtained from all volunteers after a detailed explanation of all the procedures involved.

Volunteers visited the laboratory on four consecutive days and were exposed to four different environmental conditions (Table A4) inside an environmental chamber. Thermal stress was set at $30^{\circ} \mathrm{C}$ WBGT for hot and $20^{\circ} \mathrm{C}$ WBGT for temperate conditions [49]. The environmental chamber used $(2.85 \mathrm{~m} \times 2.85 \mathrm{~m} \times 4 \mathrm{~m})$ was accurate to $\pm 0.5{ }^{\circ} \mathrm{C}, \pm 3 \%$ relative humidity, $\pm 10 \mathrm{~W} / \mathrm{m}^{2}$ solar radiation, and $\pm 0.1 \mathrm{~m} / \mathrm{sec}$ wind speed within the tested range. A WBGT meter (Kestrel 5400FW, Nielsen-Kellerman, Boothwyn, PA, USA) was utilized to confirm the reliability of the simulated environment at head, chest, and feet levels. Solar radiation was measured with a solar power meter (TES 1333R, TES, Taipei, Taiwan) at head, chest, and feet level. 
Table A4. Simulated environmental conditions during lab experiments.

\begin{tabular}{ccccccc}
\hline \multirow{2}{*}{ Environment } & Thermal Stress & $\begin{array}{c}\text { AT } \\
\left({ }^{\circ} \mathbf{C}\right)\end{array}$ & $\begin{array}{c}\text { RH } \\
(\mathbf{\%})\end{array}$ & $\begin{array}{c}\text { WS } \\
(\mathbf{m} / \mathbf{s})\end{array}$ & $\begin{array}{c}\text { SR } \\
\left(\mathbf{W} / \mathbf{m}^{\mathbf{2}}\right)\end{array}$ & $\begin{array}{c}\text { WBGT } \\
\left({ }^{\circ} \mathbf{C}\right)\end{array}$ \\
\hline \multirow{2}{*}{ Outdoor } & Hot & 32.6 & 30 & 0.5 & 800 & 30 \\
\multirow{2}{*}{ Indoor } & Temperate & 19.1 & 30 & 0.5 & 800 & 20 \\
& Hot & 40.6 & 30 & 0.5 & 0 & 30 \\
& Temperate & 28.5 & 30 & 0.5 & 0 & 20 \\
\hline
\end{tabular}

$\overline{\mathrm{AT}}$ = air temperature; $\mathrm{RH}$ = relative humidity; WS = wind speed; SR = solar radiation; WBGT = wet-bulb globe temperature.

Each volunteer underwent the aforementioned four environmental scenarios in random order. To minimize participant bias in the cognitive performance and the subjective assessments used (see below), the true purpose of the study was hidden from the volunteers. Instead, they were told that the study investigated the impact of heat (not specifically solar radiation) on human performance. Once the data collection was completed, all volunteers were informed about the true purpose of the study and gave their permission to analyze and publish these data.

Each of the aforementioned four trials included a baseline assessment of cognitive performance (outside the environmental chamber; duration: approx. $20 \mathrm{~min}$ ) and three hours and twenty minutes of data collection inside the environmental chamber with three main time periods: rest (two hours), work (one hour), and recovery (twenty minutes) (Figure A4). Volunteers entered the environmental chamber five minutes prior to the start of data collection to apply and set up the equipment and sensors on their bodies.

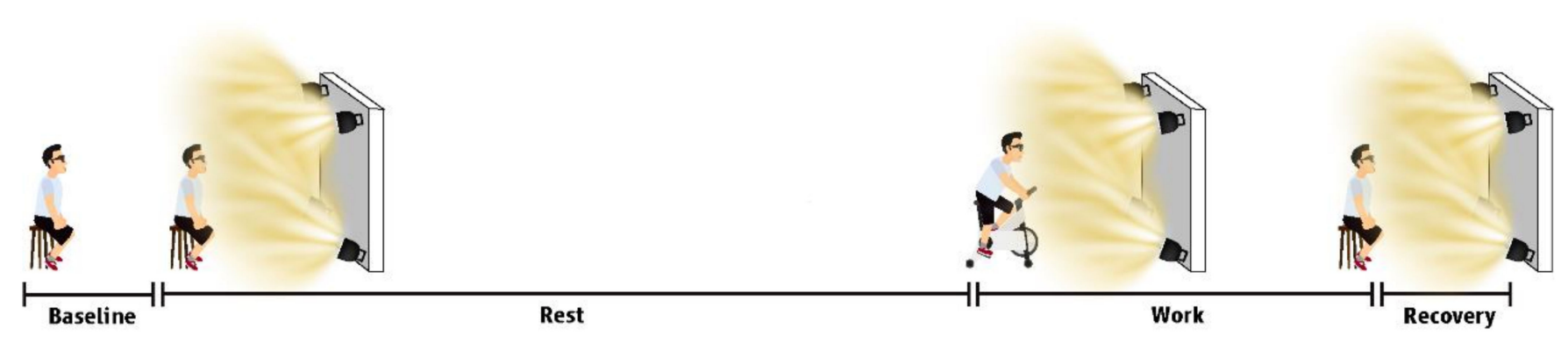

Figure A4. The experimental protocol of laboratory study.

All trials took place during the same time of the day for each participant, following an 8-h fast. Participants were requested to arrive at the laboratory in a euhydrated state and to avoid caffeine and alcohol consumption for at least twelve hours before the experiments, as well as to avoid salt and sugar consumption eight hours before the experiments. Based on existing guidelines [50], euhydration was defined as urine specific gravity $<1.020$ (urine sample taken at participants' arrival), which was assessed using a handheld refractometer (ATAGO Ltd., Tokyo, Japan). Water consumption was prohibited during the experimental protocol. The same clothing consisting of a light-blue t-shirt (100\% cotton), a black exercise pair of shorts ( $100 \%$ polyester), and a pair of medium-high socks $(100 \%$ cotton) were used by volunteers for all experiments (estimated clothing insulation $=0.38$ clo (shoes $=0.04$ clo; socks $=0.04$ clo; underwear $=0.04$ clo; t-shirt $=0.18$ clo; shorts $=0.08$ clo) $)$ [51]. Additionally, a pair of sunglasses was worn throughout the experiments.

Two days prior to the experiments, we assessed body stature (Seca 213; seca GmbH \& Co. KG; Hamburg, Germany), body mass (BC1000, Tanita corporation, Tokyo, Japan), as well as body fat and lean mass (Dual energy X-ray absorptiometry (DEXA); Lunar DPX Madison, GE Healthcare, Madison, WI, USA). During the study, continuous HR, $\mathrm{T}_{\text {core }}, \mathrm{T}_{\mathrm{sk}}$, local skin blood flow, and local sweat rate were measured. Specifically, HR was collected using wireless heart rate monitors (Polar Team2, Polar Electro Oy, Kempele, Finland). $\mathrm{T}_{\text {core }}$ was recorded using telemetric capsules (BodyCap, Caen, France). Skin 
temperature from four sites was measured using wireless thermistors (iButtons type DS1921H, Maxim/Dallas Semiconductor Corp., Sunnyvale, CA, USA) and was expressed as $\mathrm{T}_{\text {sk }}$ according to Ramanathan $\left(\mathrm{T}_{\mathrm{sk}}=0.3(\mathrm{chest}+\mathrm{arm})+0.2(\right.$ thigh + leg $)$ [37]. Skin blood flow was measured with a laser Doppler flowmeter (PeriFlux 4000, Perimed, Stockholm, Sweden) at the right forearm (brachioradialis) and leg (gastrocnemius), ensuring no direct exposure to solar radiation. The probe (PROBE 413 Integrating Probe, Perimed, Stockholm, Sweden) was held in place with a plastic holder (PH 13, Perimed, Stockholm, Sweden). Sweat rate was measured at three regions (forehead (subject to direct solar radiation), thigh/quadricep (subject to indirect solar radiation) [52], and arm/bicep (subject to indirect solar radiation)) using the ventilated capsule method. Thermal comfort ( $1=$ comfortable; 5 = extremely uncomfortable), thermal sensation $(-3=$ cold; $+3=$ hot $)$, and perceived exertion ( $6=$ no exertion at all; $20=$ maximal exertion) [53], alongside cognitive performance (see "Appendix B.3. Assessment of Cognitive Performance") were assessed at baseline, before work (at 01:40:00), and following work (at 03:00:00).

\section{Appendix B.2. Sample Size Calculation}

The minimum required sample size for investigating "differences between dependent means" was calculated using the difference in $\mathrm{T}_{\text {sk }}$ (see results of study 1 ) between workers working in indoor $\left(34.6 \pm 0.9{ }^{\circ} \mathrm{C}\right)$ and outdoor $\left(35.8 \pm 1.0^{\circ} \mathrm{C}\right)$ conditions. Using these data, an effect size ( $\mathrm{dz}$ ) of 1.27 for the differences between indoor and outdoor conditions was computed. Assuming an $\alpha$ of 0.05 and $\beta$ of 0.90 , seven participants would provide enough power to detect a statistical difference of a similar magnitude $\left(\mathrm{G}^{*}\right.$ Power Version 3.1.9.2) [54]. Based on these calculations, a total of seven healthy individuals volunteered and were recruited for this study.

\section{Appendix B.3. Assessment of Cognitive Performance}

Familiarization for all tests was undertaken one week prior to the experiments. During these familiarizations, as well as during the data collection, volunteers were isolated in a room with no external visual and/or acoustic stimuli. Screen brightness and sound volume remained constant throughout the experiments. The same sequence of cognitive performance tests (1st: reaction time in acoustic stimuli; 2 nd: reaction time in visual stimuli; 3rd: memory test; 4th: divided attention; and 5th: vigilance) was followed throughout the experiments. Approximately 20 min were required to complete all the cognitive tests. These tests were repeated three times: at baseline (20 min before entering the chamber), before work (at 01:40:00), and following work (at 03:00:00).

- Vigilance Test: We used a well-known vigilance test described in the Test for Attentional Performance [55] that has been well-accepted in the literature [56]. To run the test, we developed a computer software (freely available at www.famelab.gr/ research/downloads / accessed on 1 July 2019). In brief, this task involves two squares arranged vertically. A pattern jumps from one square to the other. Sometimes the pattern repeats in the same square. When this happens, volunteers are instructed to touch the screen of a tablet computer as fast as possible. The total duration of this test was set to six minutes.

- Divided Attention: We used a well-known vigilance test described in the Test for Attentional Performance [55] that has been well-accepted in the literature [57]. To run the test, we developed a computer software (freely available at www.famelab. gr/research/downloads / accessed on 1 July 2019). In brief, this task involves both auditory and visual stimuli in parallel. During the test, a number of visual stimuli (crosses) appear in a random configuration in a $4 \times 4$ matrix. At the same time, volunteers hear high- and low-pitch beeps in a random order. The aim is to touch the screen of a tablet computer as fast as possible when crosses form a square and, at the same time, two high- or low-pitch beeps are emitted twice in a row.

- Memory Test: We developed a computer software (freely available at www.famelab. gr/research/downloads / accessed on 1 July 2019) to perform Sternberg's Memory 
Test [58]. In this well-known test, volunteers had to observe carefully a random sequence of one to six digits (ranging from 0 to 9) in white font and displayed for $1.2 \mathrm{~s}$ each. Following a 2 s delay, a random digit (from the numbers that were previously presented) in yellow font was presented. Participants had to indicate whether the yellow digit was part of the sequence of numbers presented or not by pressing the "yes" or "no" buttons on the screen of a tablet computer as fast as possible. Each test included a total of 24 trials.

- $\quad$ Reaction Time: We developed a computer software (freely available at www.famelab. gr/research/downloads / accessed on 1 July 2019) to assess reaction time. Participants were requested to place their finger on the screen of a tablet computer and to remove it as fast as possible after receiving a visual (i.e., screen turning from black to yellow) or acoustic (i.e., a loud beep was heard) stimulus.

\section{Appendix B.4. Work Intensity}

Volunteers were instructed to cycle at $100 \mathrm{~W}\left(52.5 \pm 3.8 \mathrm{~W} / \mathrm{m}^{2}\right)$ for $60 \mathrm{~min}$. A cycle ergometer (CycleOps 400 Pro Serie Indoor Cycle, Fitchburg, MA, USA) combined with a commercially available software Rouvy (VirtualTraining, Vimperk, Czech Republic) was used to ensure constant work intensity throughout the experiments. We adopted an absolute work intensity in W (i.e., not expressed as a function of body surface area) because that is more representative for workers who perform manual labor in occupational settings (i.e., workers with different anthropometric characteristics are expected to produce the same work output).

The metabolic rate during work was approximately $300 \mathrm{~W} / \mathrm{m}^{2}\left(297 \pm 14.1 \mathrm{~W} / \mathrm{m}^{2}\right)$, corresponding to a broad spectrum of moderate-intensity manual labor tasks, including "coal mining, drilling coal and/or $\operatorname{rock}^{\prime}\left(308.2 \mathrm{~W} / \mathrm{m}^{2}\right)$, "farming, moderate effort" $\left(279.12 \mathrm{~W} / \mathrm{m}^{2}\right)$, "fishing, commercial, moderate effort" $\left(290.75 \mathrm{~W} / \mathrm{m}^{2}\right)$, "forestry, ax chopping, slow" (290.75 W/m²), "machine tooling, operating punch press, moderate effort" $\left(290.75 \mathrm{~W} / \mathrm{m}^{2}\right)$, "shoveling, less than $10 \mathrm{lbs} / \mathrm{min}$, moderate effort" $\left(290.75 \mathrm{~W} / \mathrm{m}^{2}\right)$, "steel mill, moderate effort" ( $\left.308.2 \mathrm{~W} / \mathrm{m}^{2}\right)$, and "walking, carrying objects about 25 to $49 \mathrm{lbs}^{\text {" }}$ $\left(290.75 \mathrm{~W} / \mathrm{m}^{2}\right)$ [51,59-61]. The metabolic rate (in $\mathrm{W} / \mathrm{m}^{2}$ ) was calculated by applying body surface area [62] in an iterative method to the well-known equation developed by Fiala [51,63].

\section{Appendix B.5. Solar Radiation}

The amount of radiant heat absorbed by the human body during typical outdoor work varies considerably based on body posture [64]. A previous study identified that $81.4 \%$ of the work shift time is spent crouching, $12 \%$ standing, and $6.6 \%$ sitting [51]. Based on this information, the participants were placed in a crouching position throughout the data collection: sitting on a high stool during rest and recovery, and cycling during work. The solar radiation level was set at $800 \mathrm{~W} / \mathrm{m}^{2}$, which is a typical level of solar radiation during work under clear sky conditions [13]. Solar radiation was simulated by four Compact Source Iodide lamps previously tested and used for this purpose [65]. These lamps are characterized by high light efficacy $(>90 \mathrm{~lm} / \mathrm{W})$ and good balance in spectral qualities (the mean difference between solar radiation and these lamps was found to be just $0.01 \%$ across six spectral bands), and thus they are capable of simulating sun light [65]. Two lamps were located at the height of $2.2 \mathrm{~m}$, facing participants at about $50^{\circ}$ angle (more representative for the average sun angle). The other two lamps were located $50 \mathrm{~cm}$ above the ground to ensure equal distribution of radiation waves on the participants bodies. Interindividual differences in body stature between participants were addressed by personalized adjustments in the angle and intensity (using a potentiometer) of the lamps.

\section{Appendix B.6. Statistical Analysis}

Effect sizes were calculated to investigate the differences in cognitive performance and physiological parameters between outdoor hot and indoor hot, as well as between 
outdoor temperate and indoor temperate environments (Table A5). The magnitude of effect sizes was determined as follows: $d(0.01)=$ very small; $d(0.2)=$ small; $d(0.5)=$ medium; $\mathrm{d}(0.8)=$ large; $\mathrm{d}(1.2)=$ very large; and $\mathrm{d}(2.0)=$ huge [66]. Pearson's correlation coefficient $\mathrm{r}$ was used to examine the relationships between mean skin temperature, core temperature, and cognitive performance. A Bonferroni adjusted alpha of 0.006 was set for detecting statistically significant associations for this set of correlation analyses. Statistical analyses were conducted using both the SPSS v25.0 (IBM, Armonk, NY, USA) and Excel spreadsheets (Microsoft Office, Microsoft Corp., Redmond, WA, USA). The level of significance for these analyses was set at $p<0.05$ unless otherwise specified.

Appendix B.7. Results

Table A5. The effect of solar radiation on physiological responses and cognitive performance.

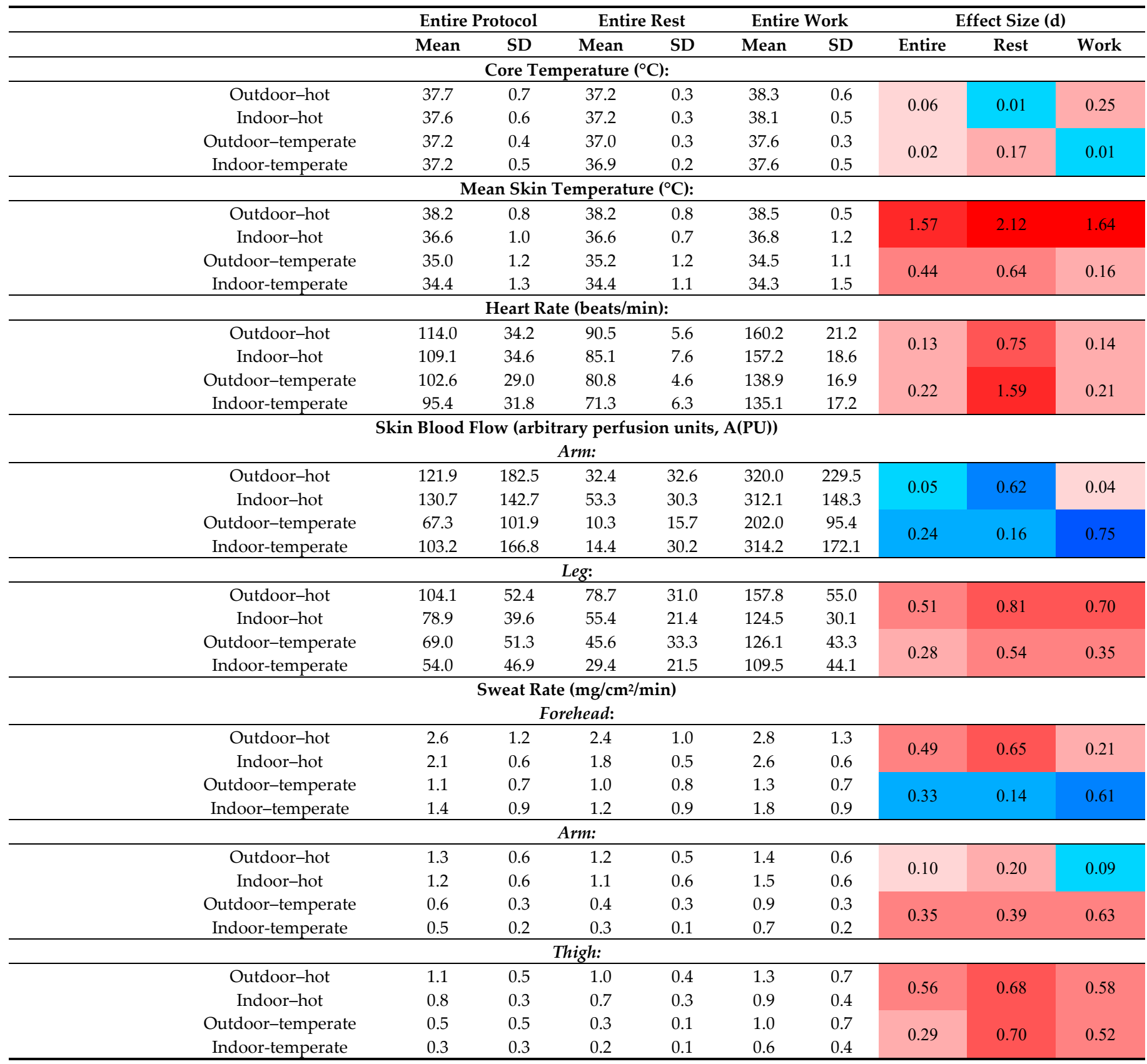


Table A5. Cont.

\begin{tabular}{|c|c|c|c|c|c|c|c|c|c|}
\hline & \multicolumn{2}{|c|}{ Entire Protocol } & \multicolumn{2}{|c|}{ Entire Rest } & \multicolumn{2}{|c|}{ Entire Work } & \multicolumn{3}{|c|}{ Effect Size (d) } \\
\hline & Mean & SD & Mean & SD & Mean & SD & Entire & Rest & Work \\
\hline \multicolumn{10}{|c|}{ Divided Attention (\% Mistakes): } \\
\hline Outdoor-hot & 30.4 & 21.0 & 29.0 & 26.6 & 31.8 & 15.5 & \multirow{2}{*}{0.47} & \multirow{2}{*}{0.49} & \multirow{2}{*}{0.45} \\
\hline Indoor-hot & 21.0 & 15.9 & 17.4 & 15.4 & 24.0 & 16.8 & & & \\
\hline Outdoor-temperate & 17.9 & 15.6 & 15.1 & 18.1 & 20.7 & 13.5 & \multirow{2}{*}{0.12} & \multirow{2}{*}{0.02} & \multirow{2}{*}{0.21} \\
\hline Indoor-temperate & 16.1 & 13.2 & 14.7 & 12.4 & 17.5 & 14.9 & & & \\
\hline \multicolumn{10}{|c|}{ Vigilance (\% Mistakes): } \\
\hline Outdoor-hot & 14.6 & 12.7 & 13.5 & 11.6 & 15.9 & 14.8 & \multirow{2}{*}{0.48} & \multirow{2}{*}{1.18} & \multirow{2}{*}{0.14} \\
\hline Indoor-hot & 8.7 & 10.1 & 2.7 & 3.2 & 13.9 & 11.2 & & & \\
\hline Outdoor-temperate & 10.8 & 15.7 & 9.7 & 15.0 & 11.8 & 17.6 & \multirow{2}{*}{0.44} & \multirow{2}{*}{0.35} & \multirow{2}{*}{0.50} \\
\hline Indoor-temperate & 5.1 & 6.0 & 5.2 & 7.2 & 4.9 & 5.0 & & & \\
\hline \multicolumn{10}{|c|}{ Memory test (\% Mistakes): } \\
\hline Outdoor-hot & 8.7 & 6.2 & 8.3 & 7.6 & 9.0 & 4.9 & \multirow{2}{*}{0.21} & \multirow{2}{*}{0.56} & \multirow{2}{*}{0.12} \\
\hline Indoor-hot & 7.1 & 8.1 & 4.2 & 5.9 & 10.0 & 9.6 & & & \\
\hline Outdoor-temperate & 4.5 & 5.0 & 3.0 & 4.6 & 6.0 & 5.3 & \multirow{2}{*}{0.09} & 0 & (15 \\
\hline Indoor-temperate & 5.0 & 5.5 & 5.8 & 8.1 & 4.2 & 0.0 & & 0.40 & 0.45 \\
\hline & & tory $\mathrm{R}$ & tion Ti & (ms): & & & & & \\
\hline Outdoor-hot & 527.0 & 71.0 & 517.9 & 80.0 & 536.1 & 65.7 & 035 & 030 & 044 \\
\hline Indoor-hot & 501.2 & 64.0 & 491.3 & 85.4 & 511.1 & 37.0 & 0.35 & 0.30 & 0.44 \\
\hline Outdoor-temperate & 474.5 & 54.5 & 475.6 & 61.5 & 473.4 & 51.4 & 0.04 & & \\
\hline Indoor-temperate & 477.6 & 93.8 & 486.3 & 63.5 & 468.9 & 121.8 & 0.04 & 0.16 & 0.04 \\
\hline & & ual $\mathrm{Re}$ & on Tim & ns): & & & & & \\
\hline Outdoor-hot & 214.7 & 25.7 & 216.6 & 26.5 & 212.9 & 26.8 & 012 & 032 & 005 \\
\hline Indoor-hot & 211.1 & 28.7 & 207.7 & 25.1 & 214.4 & 33.5 & 0.12 & 0.32 & 0.05 \\
\hline Outdoor-temperate & 218.6 & 26.5 & 227.4 & 32.1 & 209.7 & 17.8 & 027 & 011 & 0.44 \\
\hline Indoor-temperate & 236.7 & 84.9 & 223.3 & 34.6 & 250.1 & 118.4 & 0.27 & 0.11 & 0.44 \\
\hline & erceive & ertion & = none; & $=\operatorname{maxi}$ & & & & & \\
\hline Outdoor-hot & 11.9 & 4.3 & 9.6 & 2.6 & 16.9 & 2.3 & 足00 & 000 & 015 \\
\hline Indoor-hot & 11.5 & 4.5 & 9.3 & 3.1 & 16.5 & 2.7 & 0.09 & 0.08 & 0.15 \\
\hline Outdoor-temperate & 9.5 & 2.6 & 8.1 & 1.5 & 12.3 & 2.2 & & & \\
\hline Indoor-temperate & 8.9 & 2.5 & 7.4 & 1.3 & 11.8 & 1.8 & 0.22 & 0.46 & 0.23 \\
\hline & ermal S & tion ( & very cc & $+3=v$ & hot): & & & & \\
\hline Outdoor-hot & 2.5 & 0.6 & 2.4 & 0.6 & 2.8 & 0.4 & 000 & 008 & 018 \\
\hline Indoor-hot & 2.5 & 0.5 & 2.4 & 0.5 & 2.8 & 0.5 & 0.00 & 0.08 & 0.18 \\
\hline Outdoor-temperate & 1.1 & 0.8 & 0.9 & 0.6 & 1.6 & 0.9 & 070 & 109 & 052 \\
\hline Indoor-temperate & 0.6 & 0.7 & 0.3 & 0.5 & 1.2 & 0.6 & 0.70 & 1.09 & 0.52 \\
\hline & 1 Comfo & $=\mathrm{com}$ & table; 5 & ery un & ffortabl & & & & \\
\hline Outdoor-hot & 3.2 & 1.0 & 2.9 & 0.9 & 4.0 & 0.9 & & & \\
\hline Indoor-hot & 3.4 & 0.8 & 3.1 & 0.7 & 4.2 & 0.7 & 0.20 & 0.26 & 0.25 \\
\hline Outdoor-temperate & 2.2 & 0.8 & 1.9 & 0.5 & 2.8 & 0.7 & $05 ?$ & 070 & 048 \\
\hline Indoor-temperate & 1.8 & 0.8 & 1.4 & 0.5 & 2.4 & 0.8 & 0.52 & 0.19 & 0.48 \\
\hline
\end{tabular}

Notes:

Positive values correspond to an incremental effect of solar radiation on this parameter.

Negative values correspond to a diminishing effect of the solar radiation on this parameter. Effect size values were grouped to the closest category.

\begin{tabular}{ccccccc}
\hline Effect Size (d): & & & & & \\
\hline & Very Small & Small & Medium & Large & Very Large & Huge \\
\hline positive & 0.01 & 0.2 & 0.5 & 0.8 & 1.2 & 2.0 \\
negative & 0.01 & 0.2 & 0.5 & 0.8 & 1.2 & 2.0 \\
\hline
\end{tabular}


Table A6. Associations between cognitive performance and either the mean skin temperature or core temperatures. ${ }^{*}$ indicates statistically significant association at a Bonferroni adjusted alpha of 0.006 .

\begin{tabular}{ccccc}
\hline Parameter & \multicolumn{2}{c}{$\begin{array}{c}\text { Mean Skin } \\
\text { Temperature }\end{array}$} & \multicolumn{2}{c}{ Core Temperature } \\
& $\mathbf{r}$ & $p$ & $\mathbf{r}$ & $p$ \\
\hline Vigilance (more mistakes) & 0.137 & 0.229 & 0.297 & 0.008 \\
Divided Attention (more mistakes) & 0.248 & 0.027 & 0.274 & 0.015 \\
Memory (more mistakes) & 0.373 & 0.002 & 0.335 & $0.006^{*}$ \\
Perceived Exertion (worse) & 0.333 & 0.003 & 0.804 & $<0.001^{*}$ \\
Thermal Sensation (worse) & 0.562 & $<0.001$ & 0.523 & $<0.001^{*}$ \\
Thermal Comfort (worse) & 0.467 & $<0.001$ & 0.671 & $<0.001^{*}$ \\
Reaction time in auditory stimuli (slower) & 0.377 & 0.001 & 0.364 & $0.001^{*}$ \\
Reaction time in visual stimuli & 0.18 & 0.873 & -0.025 & 0.873 \\
\hline
\end{tabular}

\section{Appendix C}

Study 3. Identifying factors increasing the adverse effects of sun exposure experienced by agriculture and construction workers.

\section{Appendix C.1. Experimental Protocol}

The experimental protocol for these field experiments was approved by the National Bioethical Review Board of Cyprus (protocol number: EEBK EP 2017.01.61) in accordance with the Declaration of Helsinki. An observational study was conducted in Cyprus to identify possible factors affecting the radiant heat exchange (i.e., between workers' bodies and the surrounding environment) leading to increased heat strain in outdoor occupational settings. For this reason, 78 agriculture workers (112 full work shifts) from seven countries (Bangladesh, Cyprus, Egypt, India, Philippines, Romania, and Vietnam) were monitored over a period of three months. Specifically, video recordings (Hero 5 black, GoPro, San Mateo, CA, USA) were used to examine workers' clothing during actual work shifts performed outdoors. Munsell color system $(0=$ black to $10=$ white $)$ was used to categorize workers' clothing (dark color $=0$ to 5 and light color $=6$ to 10) [67]. To minimize examiner bias, an examination of workers' clothing was conducted independently by two investigators. Any conflicts were resolved by discussion.

\section{Appendix C.2. Results}

Table A7. Anthropometric characteristics of the participants in Study 4.

\begin{tabular}{lcccc}
\hline & Mean & SD & Minimum & Maximum \\
\hline & \multicolumn{3}{c}{ Agriculture } \\
\hline Age (years) & 39.2 & 11.8 & 21.0 & 56.0 \\
Body mass $(\mathrm{kg})$ & 77.0 & 16.2 & 54.2 & 100.5 \\
Body stature $(\mathrm{m})$ & 1.68 & 0.09 & 1.53 & 1.81 \\
Body mass index $\left(\mathrm{kg} / \mathrm{m}^{2}\right)$ & 27.1 & 5.2 & 20.9 & 36.5 \\
\hline & \multicolumn{5}{c}{ Construction } \\
\hline Age (years) & 33.6 & 8.4 & 18.0 & 52.0 \\
Body mass $(\mathrm{kg})$ & 65.4 & 8.6 & 50.0 & 90.0 \\
Body stature $(\mathrm{m})$ & 1.66 & 0.06 & 1.54 & 1.80 \\
Body mass index $\left(\mathrm{kg} / \mathrm{m}^{2}\right)$ & 23.9 & 3.1 & 17.3 & 33.4 \\
\hline
\end{tabular}

\section{Appendix D}

Study 4. Interventions to mitigate the sunlight-induced heat strain experienced by workers who work in agriculture and construction. 


\section{Appendix D.1. Experimental Protocol}

The experimental protocol (ClinicalTrials.gov (accessed on 1 July 2019) ID: NCT04160728) for these field experiments was approved by the Bioethical Committee of the School of Exercise Science of the University of Thessaly (protocol number: 1217) and the National Bioethical Review Board of Cyprus (protocol number: EEBK EP 2017.01.61) in accordance with the Declaration of Helsinki. Two experimental field studies were conducted in Cyprus (agriculture workers) and Qatar (construction workers) to investigate whether providing workers with light-colored clothes is able to mitigate the sunlight-induced heat strain they experience during their work shifts. The idea behind this study was to find a feasible and economically viable mitigation strategy able to help workers in an occupational setting.

\section{Appendix D.2. Intervention in Agriculture}

A group of six (see "Sample Size Calculation") agriculture workers (performing tasks at $\sim 200 \mathrm{~W} / \mathrm{m}^{2}$ ) from Cyprus volunteered to participate in the study. The study involved monitoring two consecutive full work shifts ("business as usual" and "white clothing" scenarios). All testing procedures were similar between the two scenarios, with the only difference being that workers during the white clothing scenario were provided with white hats and t-shirts (all 100\% cotton), as well as they were instructed to wear light-colored pants. One day prior to the start of data collection, volunteers underwent a familiarization session that included information regarding all data collection procedures. Written informed consent was obtained from all volunteers prior to their participation in the study.

\section{Appendix D.3. Intervention in Construction}

A group of 41 (see "Sample Size Calculation") construction workers (performing tasks at $\sim 100 \mathrm{~W} / \mathrm{m}^{2}$ ) from Qatar volunteered to participate in the study. The study involved monitoring two consecutive full work shifts ("business as usual" and "white coverall" scenarios). All testing procedures were similar between the two scenarios, with the only difference being that workers during the white coverall (half: 100\% cotton; half: $65 \%$ cotton and $35 \%$ polyester) scenario were provided with white coveralls (estimated clothing insulation $=0.91$ clo (shoes $=0.04$ clo; socks $=0.04$ clo; underwear $=0.04$ clo; t-shirt $=0.18$ clo; coverall $=0.61 \mathrm{clo})$ ) $[12,51]$. One week prior to the start of data collection, volunteers underwent a familiarization session that included information regarding all data collection procedures. Written informed consent was obtained from all volunteers prior to their participation in the study.

\section{Appendix D.4. Data Collection}

Anthropometric data (age; body stature (Seca 213; seca GmbH \& Co. KG; Hamburg, Germany) and body mass (BC1000, Tanita Corporation, Tokyo, Japan)) were collected two days prior to the experiment. During the study, continuous heart rate, $\mathrm{T}_{\text {core }}$ and $\mathrm{T}_{\mathrm{sk}}$ data were collected using wireless heart rate monitors (Polar Team2. Polar Electro Oy, Kempele, Finland), telemetric capsules (BodyCap, Caen, France), and wireless thermistors (iButtons type DS1921H, Maxim/Dallas Semiconductor Corp., Sunnyvale, CA, USA), respectively. Skin temperature data were collected from four sites (chest, arm, thigh, and lower leg) and were expressed as $\mathrm{T}_{\mathrm{sk}}\left(\mathrm{T}_{\mathrm{sk}}=0.3(\right.$ chest + arm $)+0.2($ thigh + leg $\left.)\right)$ [37]. Furthermore, continuous environmental data (WBGT and solar radiation) were collected using a portable weather station (Kestrel 5400FW, Nielsen-Kellerman, Boothwyn, PA, USA) and a handheld solar power meter (TES 1333R, TES, Taipei, Taiwan), respectively. Video recordings (Hero 5 black, GoPro, San Mateo, CA, USA) were used to calculate work intensity by means of time-motion analysis in agriculture [13], while real-time task analysis was utilized to evaluate labor intensity in construction. 


\section{Appendix D.5. Sample Size Calculation}

The minimum required sample size for investigating "differences between dependent means" was calculated using the difference in $\mathrm{T}_{\text {sk }}$ (see results of study 2; Table A1) between Outdoor-Heat $\left(38.2 \pm 0.8^{\circ} \mathrm{C}\right)$ and Indoor-Heat $\left(36.6 \pm 1.0^{\circ} \mathrm{C}\right)$ environmental scenarios. Using these data, an effect size (dz) of 1.88 for the differences between Outdoor-Heat and Indoor-Heat environmental scenarios was computed. Assuming an $\alpha$ of 0.05 and $\beta$ of 0.95 , five participants would provide enough power to detect a statistical difference of a similar magnitude ( $G^{*}$ Power Version 3.1.9.2) [54]. Based on these calculations, a total of six healthy individuals volunteered and were recruited for the interventions conducted in agriculture, while 41 more individuals volunteered for the interventions in the construction sector.

\section{Appendix D.6. Statistical Analysis}

Effect sizes were calculated to examine possible differences in the physiological heat strain experienced by the workers between "business as usual" and "white clothing" scenarios. The magnitude of effect sizes was determined as follows: $d(0.01)=$ very small; $\mathrm{d}(0.2)=$ small; $\mathrm{d}(0.5)=$ medium; $\mathrm{d}(0.8)=$ large; $\mathrm{d}(1.2)=$ very large; and $\mathrm{d}(2.0)=$ huge [66] Statistical analyses were conducted using Excel spreadsheets (Microsoft Office, Microsoft Corp., Redmond, WA, USA).

\section{Appendix D.7. Results}

Table A8. Differences in workers' physiological responses between "business as usual" and "white clothing" scenarios.

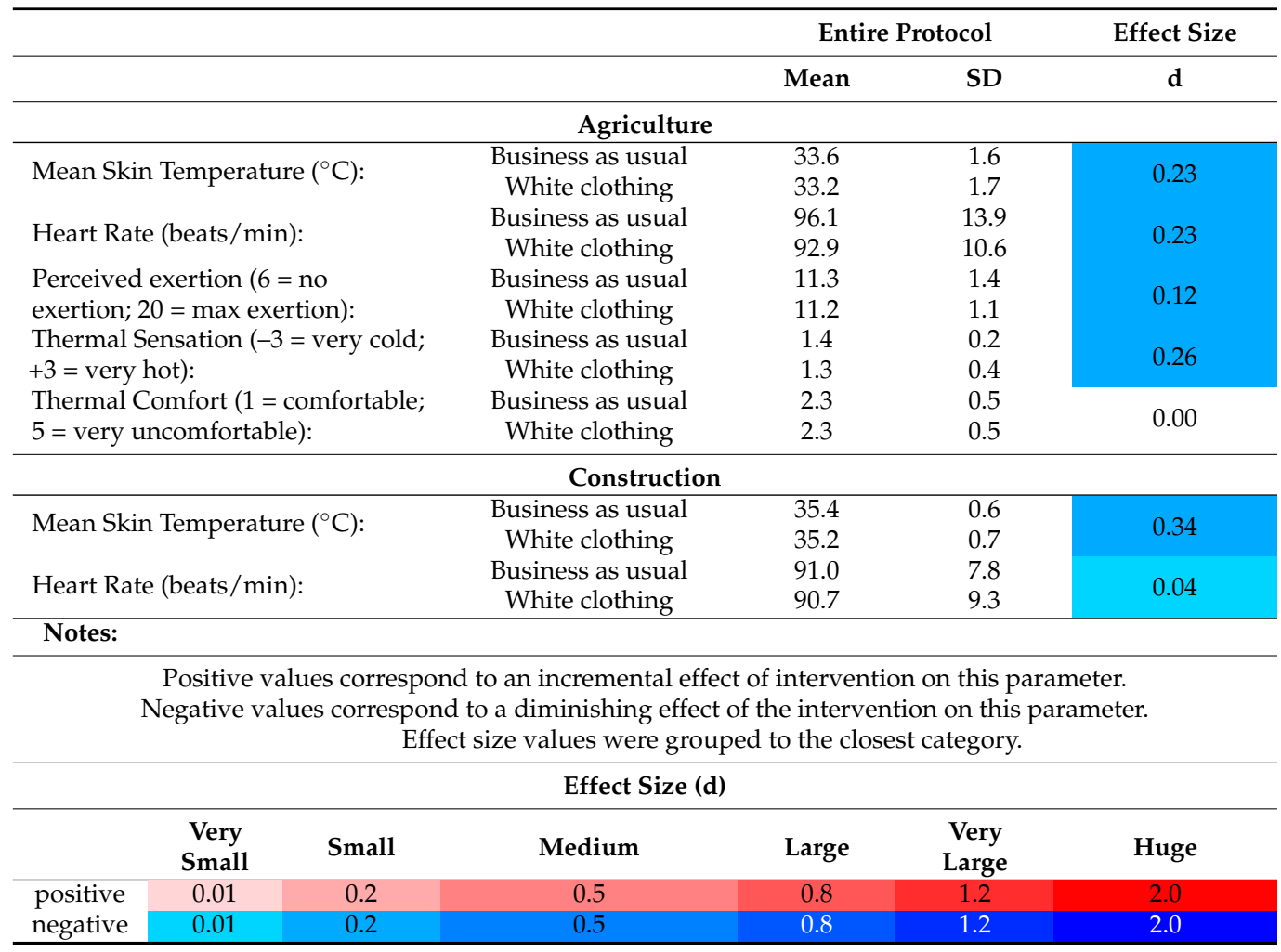

\section{References}

1. Piil, J.F.; Christiansen, L.; Morris, N.B.; Mikkelsen, C.J.; Ioannou, L.G.; Flouris, A.D.; Lundbye-Jensen, J.; Nybo, L. Direct exposure of the head to solar heat radiation impairs motor-cognitive performance. Sci. Rep. 2020, 10, 7812. [CrossRef]

2. Intergovernmental Panel on Climate Change. Climate Change 2014-Impacts, Adaptation, and Vulnerability: Part A: Global and Sectoral Aspects; Cambridge University Press: Cambridge, UK, 2014.

3. International Labour Organization. Working on A Warmer Planet: The Impact of Heat Stress on Labour Productivity and Decent Work; International Labour Organization: Geneva, Switzerland, 2019. 
4. Morris, N.B.; Jay, O.; Flouris, A.D.; Casanueva, A.; Gao, C.; Foster, J.; Havenith, G.; Nybo, L. Sustainable solutions to mitigate occupational heat strain-An umbrella review of physiological effects and global health perspectives. Environ. Health 2020, 19, 95. [CrossRef] [PubMed]

5. Food and Agriculture Organization of the United Nations (FAO) Decent Rural Employment: Key for Poverty Reduction and Food Security; Food and Agriculture Organization of the United Nations (FAO): Rome, Italy, 2010.

6. DARA: Climate Vulnerable Forum Climate Vulnerability Monitor. A Guide to the Cold Calculus of a Hot Planet, 2nd ed.; International Environment House: Geneva, Switzerland, 2012.

7. Flouris, A.D.; Kenny, G.P. Heat remains unaccounted for in thermal physiology and climate change research. F1000Research 2017, 6. [CrossRef]

8. Intergovernmental Panel on Climate Change. Summary for Policymakers; World Meteorological Organization: Geneva, Switzerland, 2018; p. 22.

9. Grandi, C.; Borra, M.; Militello, A.; Polichetti, A. Impact of climate change on occupational exposure to solar radiation. Ann. Dell'istituto Super. Sanita 2016, 52, 343-356. [CrossRef]

10. Flouris, A.D.; Dinas, P.C.; Ioannou, L.G.; Nybo, L.; Havenith, G.; Kenny, G.P.; Kjellstrom, T. Workers' health and productivity under occupational heat strain: A systematic review and meta-analysis. Lancet Planet. Health 2018, 2, e521-e531. [CrossRef]

11. Flouris, A.D.; Ioannou, L.G.; Dinas, P.C.; Mantzios, K.; Gkiata, P.; Gkikas, G.; Vliora, M.; Amorim, T.; Tsoutsoubi, L.; Kapnia, A.; et al. Assessment of Occupational Heat Strain and Mitigation Strategies in Qatar; International Labour Organization: Geneva, Switzerland, 2019.

12. Ioannou, L.G.; Mantzios, K.; Tsoutsoubi, L.; Panagiotaki, Z.; Kapnia, A.K.; Ciuha, U.; Nybo, L.; Flouris, A.D.; Mekjavic, I.B. Effect of a Simulated Heat Wave on Physiological Strain and Labour Productivity. Int. J. Environ. Res. Public Health 2021, $18,3011$. [CrossRef]

13. Ioannou, L.G.; Tsoutsoubi, L.; Samoutis, G.; Bogataj, L.K.; Kenny, G.P.; Nybo, L.; Kjellstrom, T.; Flouris, A.D. Time-motion analysis as a novel approach for evaluating the impact of environmental heat exposure on labor loss in agriculture workers. Temperature 2017, 4, 1-11. [CrossRef]

14. McPherson, M.J. Physiological reactions to climatic conditions. In Subsurface Ventilation and Environmental Engineering; Springer: Berlin, Germany, 1993; pp. 603-650.

15. Wyndham, C. Role of skin and of core temperatures in man's temperature regulation. J. Appl. Physiol. 1965, 20, 31-36. [CrossRef]

16. Dehghan, H.; Mortazavi, S.B.; Jafari, M.J.; Meraci, M.R.; Khavanin, A.L.I.; Jahangiri, M. Construct validation of a heat strain score index with structural equation modeling. Health Syst. Res. 2011, 6, 601-612.

17. Flouris, A.D.; Schlader, Z.J. Human behavioral thermoregulation during exercise in the heat. Scand. J. Med. Sci. Sports 2015, 25, 52-64. [CrossRef]

18. Flouris, A.D. Functional architecture of behavioural thermoregulation. Eur. J. Appl. Physiol. 2011, 111, 1-8. [CrossRef]

19. Flouris, A.D. Human Thermoregulation. In Heat Stress in Sport and Exercise; Springer: Cham, Switzerland, 2019 ; pp. 3-27.

20. James, C.A.; Hayes, M.; Willmott, A.G.; Gibson, O.R.; Flouris, A.D.; Schlader, Z.J.; Maxwell, N.S. Defining the determinants of endurance running performance in the heat. Temperature 2017, 4, 314-329. [CrossRef] [PubMed]

21. Junge, N.; Jørgensen, R.; Flouris, A.D.; Nybo, L. Prolonged self-paced exercise in the heat-environmental factors affecting performance. Temperature 2016, 3, 539-548. [CrossRef]

22. Romanovsky, A.A. Skin temperature: Its role in thermoregulation. Acta Physiol. 2014, 210, 498-507. [CrossRef]

23. Morris, N.B.; Piil, J.F.; Morabito, M.; Messeri, A.; Levi, M.; Ioannou, L.G.; Ciuha, U.; Pogačar, T.; Kajfež Bogataj, L.; Kingma, B.; et al. The HEAT-SHIELD project-Perspectives from an inter-sectoral approach to occupational heat stress. J. Sci. Med. Sport 2021. [CrossRef] [PubMed]

24. Flouris, A.D.; Poulianiti, K.; Bosch, P.; Jonkers, S.; Visschedijk, A.; Kjellstrom, T.; Havenith, G.; Goodwin, J.; Sotto Mayor, T.; Annaheim, S.; et al. WP2: Assessment of Health and Productivity Consequences of Current and Future Climatic Scenarios-Deliverable 2.2: Vulnerability Maps for Health and Productivity Impact across Europe; Ref. Ares(2017)5007105-13/10/2017; HEAT-SHIELD Project; European Commission: Brussels, Belgium, 2017.

25. Ioannou, L.G.; Mantzios, K.; Tsoutsoubi, L.; Nintou, E.; Vliora, M.; Gkiata, P.; Dallas, C.N.; Gkikas, G.; Agaliotis, G.; Sfakianakis, K.; et al. Occupational Heat Stress: Multi-Country Observations and Interventions. Int. J. Environ. Res. Public Health 2021, 18, 6303. [CrossRef] [PubMed]

26. Notley, S.R.; Flouris, A.D.; Kenny, G.P. On the use of wearable physiological monitors to assess heat strain during occupational heat stress. Appl. Physiol. Nutr. Metab. 2018, 43, 869-881. [CrossRef] [PubMed]

27. Varghese, B.M.; Hansen, A.; Bi, P.; Pisaniello, D. Are workers at risk of occupational injuries due to heat exposure? A comprehensive literature review. Saf. Sci. 2018, 110, 380-392. [CrossRef]

28. Clark, J.; Cena, K. Net radiation and heat transfer through clothing: The effects of insulation and colour. Ergonomics 1978, 21, 691-696. [CrossRef]

29. Gavin, T.P. Clothing and thermoregulation during exercise. Sports Med. 2003, 33, 941-947. [CrossRef]

30. International Organization for Standardization. ISO 11079: Determination \& Interpretation of Cold Stress When Using Required Clothing Insulation \& Local Cooling Effects; International Organization for Standardization: Geneva, Switzerland, 2007.

31. Nielsen, B. Solar heat load: Heat balance during exercise in clothed subjects. Eur. J. Appl. Physiol. Occup. Physiol. 1990, 60, 452-456. [CrossRef] 
32. Watanabe, S.; Horikoshi, T.; Tomita, A. Measurement of solar radiation absorptance of clothed human body in outdoor. Jpn. J. Biometeorol. 2010, 47, 165-173. [CrossRef]

33. Koppe, C.; Sari, K.; Jendritzky, G.; Menne, B. Heat-Waves: Risks and Responses; WHO Regional Office of Europe: Copenhagen, Denmark, 2004; p. 123.

34. US Centers for Disease Control \& Prevention: NIOSH. Fast Facts. Protecting Yourself from Heat Stress; Department of Health and Human Services: Cincinnati, OH, USA, 2010.

35. US Centers for Disease Control \& Prevention: NIOSH. Workplace Solution. Preventing Heat-Related Illness or Death of Outdoor Workers; Department of Health and Human Services: Cincinnati, OH, USA, 2013.

36. Zal, H.A. Recommended program for employees exposed to extremes of heat. Occup. Health Nurs. 1984, 32, 293-296. [CrossRef]

37. Ramanathan, N.L. A New Weighting System for Mean Surface Temperature of the Human Body. J. Appl. Physiol. 1964, 19, 531-533. [CrossRef]

38. Liljegren, J.C.; Carhart, R.A.; Lawday, P.; Tschopp, S.; Sharp, R. Modeling the wet bulb globe temperature using standard meteorological measurements. J. Occup. Environ. Hyg. 2008, 5, 645-655. [CrossRef] [PubMed]

39. Bongers, C.C.; Eijsvogels, T.M. Time-motion analysis in the big data era: A promising method to assess the effects of heat stress on physical performance. Temperature 2018, 5, 197-198. [CrossRef] [PubMed]

40. International Organization for Standardization. ISO 8996: Determination of Metabolic Rate; International Organization for Standardization: Geneva, Switzerland, 2004.

41. Yang, R.; Zhang, H.; You, S.; Zheng, W.; Zheng, X.; Ye, T. Study on the thermal comfort index of solar radiation conditions in winter. Build. Environ. 2019, 106456. [CrossRef]

42. Zhang, H.; Yang, R.; You, S.; Zheng, W.; Zheng, X.; Ye, T. The CPMV index for evaluating indoor thermal comfort in buildings with solar radiation. Build. Environ. 2018, 134, 1-9. [CrossRef]

43. Ely, B.R.; Cheuvront, S.N.; Kenefick, R.W.; Sawka, M.N. Aerobic performance is degraded, despite modest hyperthermia, in hot environments. Med. Sci. Sports Exerc. 2010, 42, 135-141. [CrossRef]

44. Périard, J.D.; Cramer, M.N.; Chapman, P.G.; Caillaud, C.; Thompson, M.W. Cardiovascular strain impairs prolonged self-paced exercise in the heat. Exp. Physiol. 2011, 96, 134-144. [CrossRef] [PubMed]

45. Sawka, M.N.; Cheuvront, S.N.; Kenefick, R.W. High skin temperature and hypohydration impair aerobic performance. Exp. Physiol. 2012, 97, 327-332. [CrossRef]

46. Altman, D.G. Practical Statistics for Medical Research; CRC Press: London, UK, 1990.

47. Deeks, J.J.; Higgins, J.P. Statistical algorithms in review manager 5. Stat. Methods Group Cochrane Collab. $2010,1$.

48. Andrade, C. Understanding relative risk, odds ratio, and related terms: As simple as it can get. J. Clin. Psychiatry 2015, 76, 857-861. [CrossRef]

49. International Organization for Standardization. ISO 7243: Ergonomics of the Thermal Environment-Assessment of Heat Stress Using the WBGT (Wet Bulb Globe Temperature) Index; International Organization for Standardization: Geneva, Switzerland, 2017.

50. Sawka, M.N.; Burke, L.M.; Eichner, E.R.; Maughan, R.J.; Montain, S.J.; Stachenfeld, N.S. American College of Sports Medicine position stand. Exercise and fluid replacement. Med. Sci. Sports Exerc. 2007, 39, 377-390. [CrossRef] [PubMed]

51. Ioannou, L.G.; Tsoutsoubi, L.; Mantzios, K.; Flouris, A.D. A free software to predict heat strain according to the ISO $7933: 2018$. Ind. Health 2019, 57, 711-720. [CrossRef]

52. American Society of Heating Refrigerating and Air-Conditioning Engineers. Standard 55:2004-Thermal Environmental Conditions for Human Occupancy; American Society of Heating Refrigerating and Air-Conditioning Engineers: Atlanta, GA, USA, 2004.

53. Borg, G. Psychophysical scaling with applications in physical work and the perception of exertion. Scand. J. Work Environ. Health 1990, 16, 55-58. [CrossRef] [PubMed]

54. Faul, F.; Erdfelder, E.; Lang, A.G.; Buchner, A. G*Power 3: A flexible statistical power analysis program for the social, behavioral, and biomedical sciences. Behav. Res. Methods 2007, 39, 175-191. [CrossRef] [PubMed]

55. Zimmermann, P.; Fimm, B. A test battery for attentional performance. In Applied Neuropsychology of Attention; Psychology Press: New York, NY, USA, 2004; pp. 124-165.

56. Sachs, G.; Winklbaur, B.; Jagsch, R.; Lasser, I.; Kryspin-Exner, I.; Frommann, N.; Wölwer, W. Training of affect recognition (TAR) in schizophrenia-impact on functional outcome. Schizophr. Res. 2012, 138, 262-267. [CrossRef]

57. Koenders, M.A.; Spijker, A.T.; Hoencamp, E.; Haffmans, J.P.; Zitman, F.G.; Giltay, E.J. Effects of mood state on divided attention in patients with bipolar disorder: Evidence for beneficial effects of subclinical manic symptoms. Psychiatry Res. 2014, 220, 302-308. [CrossRef]

58. Sternberg, S. High-speed scanning in human memory. Science 1966, 153, 652-654. [CrossRef]

59. Ainsworth, B.E.; Haskell, W.L.; Herrmann, S.D.; Meckes, N.; Bassett, D.R., Jr.; Tudor-Locke, C.; Greer, J.L.; Vezina, J.; Whitt-Glover, M.C.; Leon, A.S. 2011 Compendium of Physical Activities: A second update of codes and MET values. Med. Sci. Sports Exerc. 2011, 43, 1575-1581. [CrossRef]

60. Ainsworth, B.E.; Haskell, W.L.; Whitt, M.C.; Irwin, M.L.; Swartz, A.M.; Strath, S.J.; O Brien, W.L.; Bassett, D.R.; Schmitz, K.H.; Emplaincourt, P.O. Compendium of physical activities: An update of activity codes and MET intensities. Med. Sci. Sports Exerc. 2000, 32, S498-S504. [CrossRef] [PubMed]

61. Poulianiti, K.P.; Havenith, G.; Flouris, A.D. Metabolic energy cost of workers in agriculture, construction, manufacturing, tourism, and transportation industries. Ind. Health 2018, 57, 283-305. [CrossRef] [PubMed] 
62. Du Bois, D.; Du Bois, E.F. Clinical calorimetry: Tenth paper a formula to estimate the approximate surface area if height and weight be known. Arch. Intern. Med. 1916, 17, 863-871. [CrossRef]

63. Fiala, D. Dynamic Simulation of Human Heat Transfer and Thermal Comfort. Ph.D. Thesis, De Montfort University, Leicester, UK, 1998.

64. Ward, E.; Underwood, C. The effect of posture on the solar radiation area of man. Ergonomics 1967, 10, 399-409. [CrossRef]

65. Beeson, E. The CSI lamp as a source of radiation for solar simulation. Lighting Res. Technol. 1978, 10, 164-166. [CrossRef]

66. Sawilowsky, S.S. New effect size rules of thumb. J. Mod. Appl. Stat. Methods 2009, 8, 26. [CrossRef]

67. Cooper, F.G. Munsell Manual of Color: Defining and Explaining the Fundamental Characteristics of Color; Munsell Color Company, Inc.: Baltimore, MD, USA, 1929. 